Article

\title{
Extended Simulation Function via Rational Expressions
}

\author{
Rawan Alsubaie ${ }^{1}$, Badr Alqahtani ${ }^{1}$, Erdal Karapınar ${ }^{2,3, *}$ [D \\ and Antonio Francisco Roldán López de Hierro $4, * \mathbb{D}$ \\ 1 Department of Mathematics, King Saud University, P.O. Box 2455, Riyadh 11451, Saudi Arabia; \\ rawansaud.sss@gmail.com (R.A.); balqahtani1@ksu.edu.sa (B.A.) \\ 2 Department of Medical Research, China Medical University Hospital, China Medical University, \\ Taichung 40402, Taiwan \\ 3 Department of Mathematics, Çankaya University, 06790 Etimesgut, Ankara, Turkey \\ 4 Department of Statistics and Operations Research, University of Granada, 18010 Granada, Spain \\ * Correspondence: erdalkarapinar@yahoo.com (E.K.); aroldan@ugr.es (A.F.R.L.d.H.)
}

Received: 27 March 2020; Accepted: 21 April 2020; Published: 3 May 2020

\begin{abstract}
In this paper, we introduce some common fixed point theorems for two distinct self-mappings in the setting of metric spaces by using the notion of a simulation function introduced in 2015. The contractivity conditions have not to be verified for all pairs of points of the space because it is endowed with an antecedent conditions. They are also of rational type because the involved terms in the contractivity upper bound are expressed, in some cases, as quotients.
\end{abstract}

Keywords: fixed point; metric space; simulation function; rational expression; common fixed point

PACS: 46T99; 47H10; 54H25

\section{Introduction and Preliminaries}

The notion of a simulation function, introduced by Khojasteh, Shukla and Radenović [1] in 2015, has become into one of the most studied concepts in the field of Fixed Point Theory in recent times. To support this affirmation, we refer the reader to the following recent papers: [2-5] The great success of simulation functions has inspired many other developments (see, e.g., [6-11]), even in the fuzzy setting (see, e.g., [12]). In this manuscript, we use the notion of simulation function in order to prove two common fixed point theorems involving two distinct self-mappings. The importance of these results can be evaluated through the great number of possible consequences that it can easily be derived from the main theorems.

Throughout the manuscript, let $(X, d)$ be a metric space. We denote $\mathbb{N}_{0}:=\mathbb{N} \cup\{0\}$ where $\mathbb{N}$ is the set of all positive integers. Further, $\mathbb{R}$ represents the real numbers and $\mathbb{R}_{0}^{+}:=[0,+\infty)$. Background on Fixed Point theory can be found on [13].

Definition 1. ([1]) Let $\eta:[0,+\infty) \times[0,+\infty) \rightarrow \mathbb{R}$ be a mapping, then $\eta$ is called a simulation function if it satisfies the following conditions:

( $\left.\eta_{i i}\right) \eta(t, s)<s-t$ for all $s, t>0$.

$\left(\eta_{i i i}\right)$ if $\left\{t_{n}\right\},\left\{s_{n}\right\}$ are sequences in $(0,+\infty)$ such that

$$
\lim _{n \rightarrow \infty} t_{n}=\lim _{n \rightarrow \infty} s_{n}>0 \text { implies } \limsup _{n \rightarrow \infty} \eta\left(t_{n}, s_{n}\right)<0 .
$$

Example 1. We recall some examples of simulation functions given in [1].

$\left(a_{1}\right)$ For each $s, t \geq 0$, let $\eta(t, s)=\alpha s-t$, where $\alpha \in[0,1)$. 
$\left(a_{2}\right)$ For each $s, t \geq 0$, let $\eta(t, s)=\phi(s)-t$, where $\phi:[0,+\infty) \rightarrow[0,+\infty)$ is a mapping such that for each $s>0, \phi(s)<s$ and $\lim \sup _{t \rightarrow s} \phi(t)<s$.

$\left(a_{3}\right)$ For each $s, t \geq 0$, let $\eta(t, s)=s \phi(s)-t$, where $\phi:[0,+\infty) \rightarrow[0,1)$ is a mapping such that for each $s>0, \limsup _{t \rightarrow s} \phi(t)<1$.

$\left(a_{4}\right)$ For each $s, t \geq 0$, let $\eta(t, s)=s-\phi(s)-t$, where $\phi:[0,+\infty) \rightarrow[0,+\infty)$ is a mapping such that for each $s>0, \liminf _{t \rightarrow s} \phi(t)>0$ or $\phi:[0,+\infty) \rightarrow[0,1)$ is a continuous function such that $\phi(t)=0$ if and only if $t=0$.

$\left(a_{5}\right)$ For each $s, t \geq 0$, let $\eta(t, s)=\psi(s)-\phi(t)$ where $\phi, \psi:[0,+\infty) \rightarrow[0,+\infty)$ are two continuous functions such that $\psi(t)=\phi(t)=0$ if and only if $t=0$ and $\psi(t)<t \leq \phi(t)$ for all $t>0$.

The set of all simulation functions is denoted by $Z$.

Theorem 1. Let $(X, d)$ be a complete metric space and let $T: X \rightarrow X$ be a Z-contraction with respect to a certain simulation function $\eta$, that is

$$
\eta(d(T x, T y), d(x, y)) \geq 0 \quad \text { for all } x, y \in X
$$

Then $T$ has a unique fixed point.

We state the following lemma which will be useful for demonstrating our main result.

Lemma 1 (See e.g., [14]). Let $(X, d)$ be a metric space and let $\left\{x_{n}\right\}$ be a sequence in $X$ such that $\lim _{n \rightarrow \infty} d\left(x_{n}, x_{n+1}\right)=0$. If $\left\{x_{2 n}\right\}$ is not a Cauchy sequence then there exists $\varepsilon>0$ and two sequences of positive integers $\left\{n_{k}\right\}$ and $\left\{m_{k}\right\}$ such that $n_{k}$ is smallest index for which $n_{k}>m_{k}>k$ and $d\left(x_{2 m_{k}}, x_{2 n_{k}}\right) \geq$ $\varepsilon$ and

1. $\lim _{k \rightarrow \infty} d\left(x_{2 m_{k}}, x_{2 n_{k}}\right)=\varepsilon$.

2. $\lim _{k \rightarrow \infty} d\left(x_{2 m_{k}-1}, x_{2 n_{k}}\right)=\varepsilon$.

3. $\lim _{k \rightarrow \infty} d\left(x_{2 m_{k}}, x_{2 n_{k}+1}\right)=\varepsilon$.

4. $\lim _{k \rightarrow \infty} d\left(x_{2 m_{k}-1}, x_{2 n_{k}+1}\right)=\varepsilon$.

Proposition 1. ([7,15]) Let $(X, d)$ be a metric space, let $T: X \rightarrow X$ be a self-mapping and let $\left\{x_{n}\right\} \subseteq X$ be a Picard sequence of $T$ (that is, $x_{n+1}=T x_{n}$ for all $n \in \mathbb{N}$ ). If $\lim _{n \rightarrow \infty} d\left(x_{n}, x_{n+1}\right)=0$, then either $\left\{x_{n}\right\}$ is almost constant or $x_{n} \neq x_{m}$ for all $n, m \in \mathbb{N}$ such that $n \neq m$.

\section{Main Results}

The symbol $\Psi$ is reserved to denote the set of all continuous and strictly increasing self-mappings $\psi$ on $[0,+\infty)$ so that $\psi(0)=0$.

Definition 2. A function $\eta:[0,+\infty) \times[0,+\infty) \rightarrow \mathbb{R}$ is called $\Psi$-simulation function if there exists $\psi \in \Psi$ so that:

$\left(\eta_{1}\right) \eta(t, s)<\psi(s)-\psi(t)$ for all $s, t>0$,

$\left(\eta_{2}\right)$ if $\left\{t_{n}\right\},\left\{s_{n}\right\}$ are sequences in $(0,+\infty)$ such that

$$
\lim _{n \rightarrow \infty} t_{n}=\lim _{n \rightarrow \infty} s_{n}>0, \text { then } \limsup _{n \rightarrow \infty} \eta\left(t_{n}, s_{n}\right)<0 \text {. }
$$

By $Z_{\Psi}$ we shall denote the set al all $\Psi$-simulation functions. Notice that the class of $\Psi$-simulation functions covers the class of simulation functions. Then, we highlight that all fixed point results obtained by simulations functions can be derived via $\Psi$-simulation functions.

Example 2. Given $\psi \in \Psi$, the following ones are examples of $\Psi$-simulation functions. For each $s, t \geq 0$, 
$\left(e_{1}\right)$ let $\eta(t, s)=\alpha \psi(s)-\psi(t)$, where $\alpha \in[0,1)$.

$\left(e_{2}\right)$ let $\eta(t, s)=\phi(\psi(s))-\psi(t)$, where $\phi:[0,+\infty) \rightarrow[0,+\infty)$ is a function such that $\phi(0)=0$, $0<\phi(s)<s$ for each $s>0$ and $\lim \sup _{t \rightarrow s} \phi(t)<s$. (for instance, $\phi(s)=\alpha s$ where $0 \leq \alpha<1$ ).

$\left(e_{3}\right)$ let $\eta(t, s)=\phi(s) \psi(s)-\psi(t)$, where $\phi:[0,+\infty) \rightarrow[0,+\infty)$ is a function such that $\lim \sup _{t \rightarrow s} \phi(t)<$ 1 for each $s>0$.

$\left(e_{4}\right)$ let $\eta(t, s)=\psi(s)-\phi(s)-\psi(t)$, where $\phi:[0,+\infty) \rightarrow[0,+\infty)$ is a function such that $\liminf _{t \rightarrow s} \phi(t)>0$ for each $s>0$.

Remark 1. Every simulation function is a $\Psi$-simulation function where $\psi$ is taken as the identity function on $[0,+\infty)$.

So all simulation functions presented in Example 1 are $\Psi$-simulation functions. However, the following example shows that there are $\Psi$-simulation function which are not simulation functions.

Example 3. Define $\eta:[0,+\infty) \times[0,+\infty) \rightarrow \mathbb{R}$ by $\eta(t, s)=2 s \phi(s)-2 t$ where $\phi: \mathbb{R} \rightarrow[0,1)$ is a mapping such that $\limsup _{t \rightarrow s} \phi(t)<1$ for each $s>0$ (for instance, $\phi(t)=\alpha$, where $0 \leq \alpha<1$ ). Then it is easy to see that $\eta$ is a $\Psi$-simulation function. However, if $\phi(s)=\alpha \in(1 / 2,1)$, then $\eta(0, s)=2 \alpha s>s$, so $\eta$ does not satisfy $\left(\eta_{i i}\right)$ and $\eta \notin Z$.

Denote by $Z_{\Psi}$ the set of all $\Psi$-simulation functions. We have just proved that:

Proposition 2. $Z \subsetneq Z_{\Psi}$.

The first of our main results is the following one.

Theorem 2. Let $(X, d)$ be a complete metric space and let $T, S: X \rightarrow X$ be two mappings such that, for all $x, y \in X$,

$$
\frac{1}{2} \min \{d(x, T x), d(y, S y)\} \leq d(x, y) \quad \text { implies } \quad \eta(d(T x, S y), m(x, y)) \geq 0,
$$

where $\eta \in Z_{\Psi}$ and, for all $x, y \in X$,

$$
m(x, y)= \begin{cases}d(T x, S x), & \text { if } x=y, \\ \max \left\{d(x, y), \frac{d(x, T x) d(y, S y)}{d(x, y)}\right\}, & \text { if } x \neq y .\end{cases}
$$

If $T$ and $S$ are continuous, then $T$ and $S$ have a unique common fixed point (that is, there is a unique $u \in X$ such that $T u=S u=u$ ). In fact, such a point is the unique fixed point of $T$ and the unique fixed point of $S$.

Proof. For the sake of clarity, we divide the proof into five steps. The first one is necessary in order to guarantee that the consecutive terms of the sequence we will construct can be supposed as distinct.

Step (1): We claim that any fixed point of $T$ or $S$ is a common fixed point of $S$ and $T$.

Suppose that $x \in X$ is a fixed point of $T$, that is, $T x=x$ (the same argument is valid if $x$ is a fixed point of $S$ ). Reasoning by contradiction, assume that $x$ is not a common fixed of $T$ and $S$. This means that $S x \neq x$. Therefore,

$$
m(x, x)=d(T x, S x)=d(x, S x)>0 .
$$

Since

$$
\frac{1}{2} \min \{d(x, T x), d(y, S y)\}=\frac{1}{2} \min \{0, d(y, S y)\}=0 \leq d(x, y),
$$

using $y=x$ in (2), we deduce that $\eta(d(T x, S x), m(x, x)) \geq 0$. In particular, taking into account that $d(x, S x)>0$ and condition $\left(\eta_{1}\right)$, we deduce that:

$$
0 \leq \eta(d(T x, S x), m(x, x))=\eta(d(x, S x), d(x, S x))<\psi(d(x, S x))-\psi(d(x, S x))=0,
$$


which is a contradiction. Therefore, we have proved that any fixed point of $T$ (or $S$ ) is a common fixed point of $S$ and $T$, so step 1 is completed.

Next, in order to conclude $T$ or $S$ has a fixed point, we define the following sequence. Starting from an arbitrary point $x_{0} \in X$, we shall built up a recursive sequence $\left\{x_{n}\right\}$ defined as:

$$
x_{2 n+1}=T x_{2 n} \quad \text { and } \quad x_{2 n+2}=S x_{2 n+1} \quad \text { for each } n \in \mathbb{N} .
$$

If there is some $n_{0} \in \mathbb{N}$ such that $x_{n_{0}+1}=x_{n_{0}}$, then $x_{n_{0}}$ is a fixed point of $T$ or $S$ (either $T x_{n_{0}}=x_{n_{0}}$ or $S x_{n_{0}}=x_{n_{0}}$ ). In this case, step 1 shows that $x_{n_{0}}$ is a fixed point of $T$ and $S$ (and it only remains to prove that this point is unique as in step 5). On the contrary case, assume, without loss of generality, that

$$
x_{k} \neq x_{k+1} \quad \text { for all } k \in \mathbb{N} .
$$

We shall consider the following set that will be useful in later stages of the proof.

$$
\mathcal{A}:=\left\{(x, y) \in X \times X: \frac{1}{2} \min \{d(x, T x), d(y, S y)\} \leq d(x, y)\right\} .
$$

Step (2): We claim that $\lim _{k \rightarrow \infty} d\left(x_{k}, x_{k+1}\right)=0$.

To prove it, at first we claim that

$$
d\left(x_{k+1}, x_{k+2}\right) \leq d\left(x_{k}, x_{k+1}\right) \quad \text { for all } k \in \mathbb{N} .
$$

To see this, suppose that $k=2 n$ for some $n \in \mathbb{N}$. We have:

$$
\begin{aligned}
\frac{1}{2} \min \left\{d\left(x_{2 n}, T x_{2 n}\right), d\left(x_{2 n+1}, S x_{2 n+1}\right)\right\} & =\frac{1}{2} \min \left\{d\left(x_{2 n}, x_{2 n+1}\right), d\left(x_{2 n+1}, x_{2 n+2}\right)\right\} \\
& \leq d\left(x_{2 n}, x_{2 n+1}\right) .
\end{aligned}
$$

So from (2) and $\left(\eta_{1}\right)$ we have:

$$
\begin{aligned}
0 & \leq \eta\left(d\left(T x_{2 n}, S x_{2 n+1}\right), m\left(x_{2 n}, x_{2 n+1}\right)\right)=\eta\left(d\left(x_{2 n+1}, x_{2 n+2}\right), m\left(x_{2 n}, x_{2 n+1}\right)\right) \\
& <\psi\left(m\left(x_{2 n}, x_{2 n+1}\right)\right)-\psi\left(d\left(x_{2 n+1}, x_{2 n+2}\right)\right)
\end{aligned}
$$

so

$$
\psi\left(d\left(x_{2 n+1}, x_{2 n+2}\right)\right)<\psi\left(m\left(x_{2 n}, x_{2 n+1}\right)\right) .
$$

Since $\psi$ is strictly increasing,

$$
d\left(x_{2 n+1}, x_{2 n+2}\right)<m\left(x_{2 n}, x_{2 n+1}\right) .
$$

On the other hand,

$$
\begin{aligned}
m\left(x_{2 n}, x_{2 n+1}\right) & =\max \left\{d\left(x_{2 n}, x_{2 n+1}\right), \frac{d\left(x_{2 n}, T x_{2 n}\right) d\left(x_{2 n+1}, S x_{2 n+1}\right)}{d\left(x_{2 n}, x_{2 n+1}\right)}\right\} \\
& =\max \left\{d\left(x_{2 n}, x_{2 n+1}\right), \frac{d\left(x_{2 n}, x_{2 n+1}\right) d\left(x_{2 n+1}, x_{2 n+2}\right)}{d\left(x_{2 n}, x_{2 n+1}\right)}\right\} \\
& =\max \left\{d\left(x_{2 n}, x_{2 n+1}\right), d\left(x_{2 n+1}, x_{2 n+2}\right)\right\} .
\end{aligned}
$$

If there is some $n_{0} \in \mathbb{N}$ such that $d\left(x_{2 n_{0}+1}, x_{2 n_{0}+2}\right) \geq d\left(x_{2 n_{0}}, x_{2 n_{0}+1}\right)$, then $m\left(x_{2 n_{0}}, x_{2 n_{0}+1}\right)=$ $d\left(x_{2 n_{0}+1}, x_{2 n_{0}+2}\right)$, which contradicts (7). 
Therefore, for each $n \in \mathbb{N}$,

$$
d\left(x_{2 n+1}, x_{2 n+2}\right)<d\left(x_{2 n}, x_{2 n+1}\right)
$$

and so

$$
m\left(x_{2 n}, x_{2 n+1}\right)=d\left(x_{2 n}, x_{2 n+1}\right) .
$$

Consequently, (6) is proved when $k \geq 0$ is an even number. By the same argument, one can check that (6) holds when $k$ is an odd number. Thus, the sequence $\left\{d\left(x_{n}, x_{n+1}\right)\right\}_{n \geq 1}$ is non-increasing and bounded from below, so it is convergent. Hence there is a real number $\gamma \geq 0$ such that

$$
\lim _{n \rightarrow \infty} d\left(x_{n}, x_{n+1}\right)=\lim _{n \rightarrow \infty} m\left(x_{n}, x_{n+1}\right)=\gamma .
$$

We claim that $\gamma=0$. To prove the claim, at first suppose that

$$
\Omega=\{(d(T x, S y), m(x, y)):(x, y) \in \mathcal{A}\} .
$$

By (2) and definition of $\mathcal{A}$, we observe that

$$
\eta(t, s) \geq 0 \quad \text { for all }(t, s) \in \Omega \backslash\{(0,0)\} .
$$

For each $n \geq 0$ we have

$$
\begin{aligned}
\frac{1}{2} \min \left\{d\left(x_{2 n}, T x_{2 n}\right), d\left(x_{2 n+1}, S x_{2 n+1}\right)\right\} & =\frac{1}{2} \min \left\{d\left(x_{2 n}, x_{2 n+1}\right), d\left(x_{2 n+1}, x_{2 n+2}\right)\right\} \\
& \leq d\left(x_{2 n}, x_{2 n+1}\right) .
\end{aligned}
$$

Thus $\left(x_{2 n}, x_{2 n+1}\right) \in \mathcal{A}$ for each $n \geq 0$. Consequently, (9) implies that

$$
\eta\left(d\left(T x_{2 n}, S x_{2 n+1}\right), m\left(x_{2 n}, x_{2 n+1}\right)\right) \geq 0 .
$$

So

$$
\limsup _{n \rightarrow \infty} \eta\left(d\left(x_{2 n+1}, x_{2 n+2}\right), m\left(x_{2 n}, x_{2 n+1}\right)\right) \geq 0 .
$$

In order to prove that $\gamma=0$, suppose, by contradiction, that $\gamma>0$. From (8) we have

$$
\left.\lim _{n \rightarrow \infty} d\left(x_{2 n+1}, x_{2 n+2}\right)=\lim _{n \rightarrow \infty} m\left(x_{2 n}, x_{2 n+1}\right)\right)=\gamma>0 .
$$

Therefore, from $\left(\eta_{2}\right)$,

$$
\limsup _{n \rightarrow \infty} \eta\left(d\left(x_{2 n+1}, x_{2 n+2}\right), m\left(x_{2 n}, x_{2 n+1}\right)\right)<0,
$$

which contradicts (10). So the claim is proved, that is,

$$
\lim _{n \rightarrow \infty} d\left(x_{n}, x_{n+1}\right)=\lim _{n \rightarrow \infty} m\left(x_{n}, x_{n+1}\right)=0 .
$$

Step (3): We claim that $\left\{x_{n}\right\}$ is a Cauchy sequence.

Since $\left\{d\left(x_{n}, x_{n+1}\right)\right\} \rightarrow 0$, Proposition 1 guarantees that $x_{n} \neq x_{m}$ for all $n, m \in \mathbb{N}$ such that $n \neq m$ (it cannot be almost constant because $x_{n} \neq x_{n+1}$ for all $n \in \mathbb{N}$ ). Reasoning by contradiction, suppose that $\left\{x_{2 n}\right\}$ is not a Cauchy sequence. Taking into account Lemma 1, there exist $\varepsilon_{0}>0$ and subsequences $\left\{x_{2 m_{k}}\right\}$ and $\left\{x_{2 n_{k}}\right\}$ of $\left\{x_{n}\right\}$ such that $n_{k}$ is the smallest index for which $n_{k}>m_{k}>k$ and $d\left(x_{2 m_{k}}, x_{2 n_{k}}\right) \geq \varepsilon_{0}$ and

$\left(l_{1}\right) \lim _{k \rightarrow \infty} d\left(x_{2 m_{k}}, x_{2 n_{k}}\right)=\varepsilon_{0}$; 
$\left(l_{2}\right) \lim _{k \rightarrow \infty} d\left(x_{2 m_{k}-1}, x_{2 n_{k}}\right)=\varepsilon_{0}$;

(l) $\lim _{k \rightarrow \infty} d\left(x_{2 m_{k}}, x_{2 n_{k}+1}\right)=\varepsilon_{0}$;

(li $) \lim _{k \rightarrow \infty} d\left(x_{2 m_{k}-1}, x_{2 n_{k}+1}\right)=\varepsilon_{0}$.

Therefore, from the definition of $m(x, y)$ we have

$$
\begin{aligned}
\lim _{k \rightarrow \infty} m\left(x_{2 n_{k}}, x_{2 m_{k}-1}\right) & =\lim _{k \rightarrow \infty} \max \left\{d\left(x_{2 n_{k}}, x_{2 m_{k}-1}\right), \frac{d\left(x_{2 n_{k}}, T x_{2 n_{k}}\right) d\left(x_{2 m_{k}-1}, S x_{2 m_{k}-1}\right)}{d\left(x_{2 n_{k}}, x_{2 m_{k}-1}\right)}\right\} \\
& =\max \left\{\varepsilon_{0}, 0\right\}=\varepsilon_{0} .
\end{aligned}
$$

So

$$
\lim _{k \rightarrow \infty} d\left(x_{2 m_{k}}, x_{2 n_{k}+1}\right)=\lim _{k \rightarrow \infty} m\left(x_{2 m_{k}-1}, x_{2 n_{k}}\right)=\varepsilon_{0}>0 .
$$

Hence, $\left(\eta_{2}\right)$ implies that

$$
\limsup _{k \rightarrow \infty} \eta\left(d\left(x_{2 m_{k}}, x_{2 n_{k}+1}\right), m\left(x_{2 m_{k}-1}, x_{2 n_{k}}\right)\right)<0 .
$$

On the other hand, we claim that for sufficiently large $k \in \mathbb{N}$, if $n_{k}>m_{k}>k$, then

$$
\frac{1}{2} \min \left\{d\left(x_{2 n_{k}}, T x_{2 n_{k}}\right), d\left(x_{2 m_{k}-1}, S x_{2 m_{k}-1}\right)\right\} \leq d\left(x_{2 n_{k}}, x_{2 m_{k}-1}\right)
$$

Indeed, since $n_{k}>m_{k}$ and $\left\{d\left(x_{n}, x_{n+1}\right)\right\}$ is non-increasing, we have

$$
\begin{aligned}
d\left(x_{2 n_{k}}, T x_{2 n_{k}}\right) & =d\left(x_{2 n_{k}}, x_{2 n_{k}+1}\right) \leq d\left(x_{2 m_{k}+1}, x_{2 m_{k}}\right) \leq d\left(x_{2 m_{k}}, x_{2 m_{k}-1}\right) \\
& =d\left(x_{2 m_{k}-1}, S x_{2 m_{k}-1}\right) .
\end{aligned}
$$

So, the left hand side of inequality (13) is equal to

$$
\frac{1}{2} d\left(x_{2 n_{k}}, T x_{2 n_{k}}\right)=\frac{1}{2} d\left(x_{2 n_{k}}, x_{2 n_{k}+1}\right) .
$$

Therefore, we must show that, for sufficiently large $k \in \mathbb{N}$, if $n_{k}>m_{k}>k$, then

$$
d\left(x_{2 n_{k}}, x_{2 n_{k}+1}\right) \leq d\left(x_{2 n_{k}}, x_{2 m_{k}-1}\right) .
$$

According to (11), there exists $k_{1} \in \mathbb{N}$ such that for any $k>k_{1}$,

$$
d\left(x_{2 n_{k}}, x_{2 n_{k}+1}\right)<\frac{\varepsilon_{0}}{2} .
$$

There also exists $k_{2} \in \mathbb{N}$ such that for any $k>k_{2}$,

$$
d\left(x_{2 m_{k}-1}, x_{2 m_{k}}\right)<\frac{\varepsilon_{0}}{2} .
$$

Hence, for any $k>\max \left\{k_{1}, k_{2}\right\}$ and $n_{k}>m_{k}>k$, we have

$$
\begin{aligned}
\varepsilon_{0} & \leq d\left(x_{2 n_{k}}, x_{2 m_{k}}\right) \leq d\left(x_{2 n_{k}}, x_{2 m_{k}-1}\right)+d\left(x_{2 m_{k}-1}, x_{2 m_{k}}\right) \\
& \leq d\left(x_{2 n_{k}}, x_{2 m_{k}-1}\right)+\frac{\varepsilon_{0}}{2} .
\end{aligned}
$$

So, one concludes that

$$
\frac{\varepsilon_{0}}{2} \leq d\left(x_{2 n_{k}}, x_{2 m_{k}-1}\right)
$$


Thus we obtain that for any $k>\max \left\{k_{1}, k_{2}\right\}$ and $n_{k}>m_{k}>k$,

$$
d\left(x_{2 n_{k}}, x_{2 n_{k}+1}\right) \leq \frac{\varepsilon_{0}}{2} \leq d\left(x_{2 n_{k}}, x_{2 m_{k}-1}\right) .
$$

Then (13) is proved. Therefore, by (2) and definition of $\mathcal{A}$, for sufficiently large $k \in \mathbb{N}$, if $n_{k}>m_{k}>k$, then $\left(x_{2 n_{k}}, x_{2 m_{k}-1}\right) \in \mathcal{A}$. Consequently, for sufficiently large $k \in \mathbb{N}$, if $n_{k}>m_{k}>k$ then

$$
\eta\left(d\left(T x_{2 n_{k}}, S x_{2 m_{k}-1}\right), m\left(x_{2 n_{k}}, x_{2 m_{k}-1}\right)\right) \geq 0 .
$$

Hence

$$
\limsup _{k \rightarrow \infty} \eta\left(d\left(x_{2 n_{k}+1}, x_{2 m_{k}}\right), m\left(x_{2 n_{k}}, x_{2 m_{k}-1}\right)\right) \geq 0,
$$

which contradicts (12). This contradiction proves that $\left\{x_{n}\right\}$ is a Cauchy sequence and, since $X$ is complete, there exists $u \in X$ such that $\left\{x_{n}\right\} \rightarrow u$ as $n \rightarrow \infty$.

Step (4): We claim that $u$ is a common fixed point of $T$ and $S$.

Since $T$ and $S$ are continuous, we deduce that

$$
u=\lim _{n \rightarrow \infty} x_{2 n+1}=\lim _{n \rightarrow \infty} T x_{2 n}=T\left(\lim _{n \rightarrow \infty} x_{2 n}\right)=T u
$$

and

$$
u=\lim _{n \rightarrow \infty} x_{2 n+2}=\lim _{n \rightarrow \infty} S x_{2 n+1}=S\left(\lim _{n \rightarrow \infty} x_{2 n+1}\right)=S u .
$$

Therefore $T u=S u=u$, that is, $u$ is a common fixed point of $T$ and $S$.

Step (5): We claim $T$ and $S$ have a unique common fixed point, and it is the unique fixed point of $T$ and the unique fixed point of $S$.

Suppose that $T$ and $S$ have two distinct common fixed points $u, v \in X$. Therefore $T u=S u=u$, $T v=S v=v$ and $d(u, v)>0$. Therefore

$$
m(u, v)=\max \left\{d(u, v), \frac{d(u, T u) d(v, S v)}{d(u, v)}\right\}=\max \{d(u, v), 0\}=d(u, v)>0 .
$$

Taking into account that

$$
\frac{1}{2} \min \{d(u, T u), d(v, S v)\}=\frac{1}{2} \min \{0,0\}=0<d(u, v),
$$

the contractivity condition (2) guarantees that $\eta(d(T u, S v), m(u, v)) \geq 0$. Therefore,

$$
0 \leq \eta(d(T u, S v), m(u, v))=\eta(d(u, v), d(u, v))<\psi(d(u, v))-\psi(d(u, v))=0,
$$

which is a contradiction. As a consequence, $T$ and $S$ have, at most, a unique common fixed point. Furthermore, if $x$ is a fixed point of $T$ or $S$, step 1 guarantees that $x$ is a common fixed point of $T$ and $S$, which is unique, so $T$ and $S$ can only have, at most, a unique fixed point.

Example 4. Let $X=[0,1]$ endowed with the usual metric $d(x, y)=|x-y|$ for all $x, y \in X$, and let $T, S: X \rightarrow X$ given by

$$
T x=\frac{x}{4} \quad \text { and } \quad S x=\frac{x}{6} \quad \text { for all } x \in X .
$$

Clearly $(X, d)$ is complete and $T$ and $S$ are continuous self-mappings on $X$. Let us show that $T$ and $S$ satisfy the hypotheses of Theorem 2 associated to the function

$$
\eta(t, s)=\frac{0.9 s-t}{2} \text { for all } t, s \in[0,+\infty)
$$


If we take $\alpha=0.9$ and $\psi(t)=t / 2$ for all $t \in[0,+\infty)$, then $\psi \in \Psi$ and

$$
\eta(t, s)=\frac{0.9 s-t}{2}=0.9 \frac{s}{2}-\frac{t}{2}=\alpha \psi(s)-\psi(t) \quad \text { for all } t, s \in[0,+\infty) .
$$

Item $\left(e_{1}\right)$ in Example 2 guarantees that $\eta \in Z_{\Psi}$. We have to prove that the contractivity condition

$$
\frac{1}{2} \min \{d(x, T x), d(y, S y)\} \leq d(x, y) \quad \text { implies } \quad \eta(d(T x, S y), m(x, y)) \geq 0
$$

holds for all $x, y \in X$. Therefore, let $x, y \in X$ be two arbitrary points such that

$$
\frac{1}{2} \min \{d(x, T x), d(y, S y)\} \leq d(x, y),
$$

and we have to prove that

$$
\eta(d(T x, S y), m(x, y)) \geq 0 .
$$

The first case occurs when $x=y$. In this case,(14) shows that $T x=x$ or $S x=x$. Clearly $T x=x$ or $S x=0$ implies that $x=0$, so

$$
\eta(d(T x, S y), m(x, y))=\eta(d(T 0, S 0), m(0,0))=\eta(0,0)=0,
$$

which means that (15) holds when $x=y$ under the assumption (14). Now assume that $x \neq y$. Notice that

$$
\begin{aligned}
& d(x, T x)=\left|x-\frac{x}{4}\right|=\frac{3 x}{4}, \quad d(y, S y)=\left|y-\frac{y}{6}\right|=\frac{5 y}{6}, \\
& d(T x, S y)=\left|\frac{x}{4}-\frac{y}{6}\right|=\frac{|3 x-2 y|}{12} .
\end{aligned}
$$

Therefore, inequality (15) is equivalent to:

$$
\begin{array}{r}
0 \leq \eta(d(T x, S y), m(x, y))=\frac{0.9 m(x, y)-d(T x, S y)}{2} \Leftrightarrow d(T x, S y) \leq 0.9 m(x, y) \\
\Leftrightarrow \quad \frac{|3 x-2 y|}{12} \leq \frac{9}{10} \max \left\{|x-y|, \frac{\frac{3 x}{4} \cdot \frac{5 y}{6}}{|x-y|}\right\}=\frac{9}{10} \max \left\{|x-y|, \frac{5 x y}{8|x-y|}\right\} .
\end{array}
$$

Since

$$
\frac{1}{2} \min \{d(x, T x), d(y, S y)\}=\frac{1}{2} \min \left\{\frac{3 x}{4}, \frac{5 y}{6}\right\}=\min \left\{\frac{3 x}{8}, \frac{5 y}{12}\right\},
$$

we will consider the cases $x<y$ and $y<x$, and the subcases depending on the number that reaches the minimum in (17).

Case (1) Suppose that $x<y$. In this case, $d(x, y)=|x-y|=y-x$.

Subcase (1.1) $\frac{3 x}{8} \leq \frac{5 y}{12}$.

In this subcase,

$$
\min \left\{\frac{3 x}{8}, \frac{5 y}{12}\right\}=\frac{3 x}{8}
$$

Hence, (14) is equivalent to

$\frac{1}{2} \min \{d(x, T x), d(y, S y)\} \leq d(x, y) \quad \Leftrightarrow \quad \frac{3 x}{8} \leq y-x \quad \Leftrightarrow \quad \frac{11 x}{8} \leq y \quad \Leftrightarrow \quad x \leq \frac{8}{11} y$.

We show that

$$
\frac{|3 x-2 y|}{12} \leq \frac{9}{10}|x-y|=\frac{9}{10}(y-x)
$$


as follows. On the one hand,

$$
\frac{3 x-2 y}{12} \leq \frac{9}{10}(y-x) \quad \Leftrightarrow \quad 15 x-10 y \leq 54 y-54 x \quad \Leftrightarrow \quad 69 x \leq 64 y \quad \Leftrightarrow \quad x \leq \frac{64}{69} y,
$$

which holds because $x \leq \frac{8}{11} y$ by (18). On the other hand,

$$
-\frac{3 x-2 y}{12} \leq \frac{9}{10}(y-x) \quad \Leftrightarrow \quad-15 x+10 y \leq 54 y-54 x \quad \Leftrightarrow \quad 39 x \leq 44 y \quad \Leftrightarrow \quad x \leq \frac{44}{39} y,
$$

which is true because $x<y$. Joining (19) and (20), we deduce that

$$
d(T x, S y)=\frac{|3 x-2 y|}{12} \leq \frac{9}{10}(y-x)=\frac{9}{10} d(x, y) \leq \frac{9}{10} m(x, y),
$$

so the contractivity condition (16) holds.

Subcase (1.2) $\frac{5 y}{12}<\frac{3 x}{8}$.

This subcase is impossible because

$$
\frac{5 y}{12}<\frac{3 x}{8} \Leftrightarrow y<\frac{36}{40} x \Leftrightarrow y<\frac{9}{10} x,
$$

which contradicts the fact that $x<y$.

Case (2) Suppose that $y<x$. In this case, $d(x, y)=|x-y|=x-y$.

Subcase (2.1) $\frac{3 x}{8}<\frac{5 y}{12}$.

In this subcase,

$$
\min \left\{\frac{3 x}{8}, \frac{5 y}{12}\right\}=\frac{3 x}{8}
$$

Notice that

$$
\frac{3 x}{8}<\frac{5 y}{12} \Leftrightarrow \frac{36 x}{40}<y \quad \Leftrightarrow \quad \frac{9}{10} x<y .
$$

This fact contradicts (14) because:

$$
\frac{3 x}{8} \leq|x-y|=x-y \quad \Leftrightarrow \quad 3 x \leq 8 x-8 y \quad \Leftrightarrow \quad 8 y \leq 5 x \quad \Leftrightarrow \quad y \leq \frac{5}{8} x,
$$

which is impossible since

$$
y \leq \frac{5}{8} x \leq \frac{9}{10} x<y
$$

Therefore, this subcase cannot hold.

Subcase (2.2) $\frac{5 y}{12} \leq \frac{3 x}{8}$.

In this case,

$$
\min \left\{\frac{3 x}{8}, \frac{5 y}{12}\right\}=\frac{5 y}{12}
$$

Therefore,

$$
\begin{gathered}
\frac{1}{2} \min \{d(x, T x), d(y, S y)\} \leq d(x, y) \quad \Leftrightarrow \quad \frac{5 y}{12} \leq x-y \\
\Leftrightarrow \quad 5 y \leq 12 x-12 y \quad \Leftrightarrow \quad y \leq \frac{12}{17} x .
\end{gathered}
$$

Since $y<x$, then $3 x-2 y=x+2(x-y)>x \geq 0$, so

$\frac{|3 x-2 y|}{12} \leq \frac{9}{10}|x-y|=\frac{9}{10}(x-y) \quad \Leftrightarrow \quad 15 x-10 y \leq 54 x-54 y \quad \Leftrightarrow \quad 44 y \leq 39 x \quad \Leftrightarrow \quad y \leq \frac{39}{44} x$, 
which holds because of (21). Hence

$$
d(T x, S y)=\frac{|3 x-2 y|}{12} \leq \frac{9}{10}(x-y)=\frac{9}{10} d(x, y) \leq \frac{9}{10} m(x, y)
$$

and the contractivity condition (16) holds.

In any case, we have proved that $\eta(d(T x, S y), m(x, y)) \geq 0$ for all $x, y \in X$ such that

$$
\frac{1}{2} \min \{d(x, T x), d(y, S y)\} \leq d(x, y) \text {. }
$$

Finally, notice that $\eta$ is not a simulation function because if $t_{0}=2$ and $s_{0}=1$, then

$$
\eta\left(t_{0}, s_{0}\right)=\frac{0.9 s_{0}-t_{0}}{2}=\frac{0.9-2}{2}=-0.55>-1=1-2=s_{0}-t_{0} .
$$

As a consequence, Theorem 2 is applicable in order to guarantee that $T$ and $S$ have a common fixed point, which is unique and, in fact, it is the unique fixed point of $T$ and the unique fixed point of S. However, other results using simulation functions are not applicable to this context.

If we pay attention to all details in Example 4, then we observe that, in fact,

$$
\frac{1}{2} \min \{d(x, T x), d(y, S y)\} \leq d(x, y) \quad \text { implies } \quad d(T x, S y) \leq \frac{9}{10} d(x, y)
$$

for all distinct points $x, y \in X$. Hence, the reader can imagine that the term

$$
\frac{d(x, T x) d(y, S y)}{d(x, y)}
$$

does not play a role in $m(x, y)$ and, indeed, in the proof. Next, we show an example in which this term is a key piece in order to guarantee that $T$ and $S$ have a common fixed point.

Example 5. Let $X=[-1,1]$ endowed with the usual metric $d(x, y)=|x-y|$ for all $x, y \in X$, and let $T, S: X \rightarrow X$ given by

$$
T x=\frac{3}{10} x \text { and } S x=-\frac{2}{5} x \quad \text { for all } x \in X .
$$

Clearly $(X, d)$ is complete and $T$ and $S$ are continuous self-mappings on $X$. We are going to show that $T$ and $S$ satisfy all the hypotheses of Theorem 2 associated to

$$
\eta(t, s)=\frac{0.9 s-t}{2} \text { for all } t, s \in[0,+\infty)
$$

Notice that in Example 4 we have proved that $\eta \in Z_{\Psi}$ but $\eta$ is not a simulation function. Before proving that $T$ and $S$ satisfy the contractivity condition, we want to highlight that condition

$$
d(T x, S y) \leq d(x, y)
$$

is false even when $x$ and $y$ are positive and they satisfy the antecedent condition in the contractivity condition. To do that, take $x_{0}=0.53$ and $y_{0}=0.73$. Then $d\left(x_{0}, y_{0}\right)=0.2$ and

$$
\begin{gathered}
\frac{1}{2} \min \left\{d\left(x_{0}, T x_{0}\right), d\left(y_{0}, S y_{0}\right)\right\}=\frac{1}{2} \min \{d(0.53,0.159), d(0.73,-0.292)\} \\
=\frac{1}{2} \min \{0.371,1.022\}=\frac{0.371}{2}=0.1855<0.2=d\left(x_{0}, y_{0}\right) .
\end{gathered}
$$


However

$$
d\left(T x_{0}, S y_{0}\right)=d(0.159,-0.292)=0.451>0.2=d\left(x_{0}, y_{0}\right)
$$

This will prove that the second term in the maximum in

$$
m(x, y)=\max \left\{d(x, y), \frac{d(x, T x) d(y, S y)}{d(x, y)}\right\}, \quad \text { when } x \neq y
$$

will be of great importance. Let $x, y \in X$ be such that

$$
\frac{1}{2} \min \{d(x, T x), d(y, S y)\} \leq d(x, y) .
$$

If $x=y$, then the previous condition guarantees that $T x=x$ or $S x=x$. This is only possible when $x=0$ and, in this case,

$$
\eta(d(T x, S y), m(x, y))=\eta(d(T 0, S 0), m(0,0))=\eta(0,0)=0 .
$$

Next, suppose that $x \neq y$. Notice that

$$
\begin{aligned}
& d(x, T x)=\left|x-\frac{3 x}{10}\right|=\frac{7|x|}{10}, \quad d(y, S y)=\left|y-\left(-\frac{2 y}{5}\right)\right|=\frac{7|y|}{5} \\
& d(T x, S y)=\left|\frac{3 x}{10}-\left(-\frac{2 y}{5}\right)\right|=\frac{|3 x+4 y|}{10}
\end{aligned}
$$

Then

$$
\frac{1}{2} \min \{d(x, T x), d(y, S y)\}=\frac{1}{2} \min \left\{\frac{7|x|}{10}, \frac{7|y|}{5}\right\}=\min \left\{\frac{7|x|}{20}, \frac{7|y|}{10}\right\} .
$$

Furthermore, the contractivity condition can be equivalently expressed as:

$$
\begin{aligned}
0 \leq \eta & (d(T x, S y), m(x, y))=\frac{0.9 m(x, y)-d(T x, S y)}{2} \\
& \Leftrightarrow \quad d(T x, S y) \leq 0.9 m(x, y) \\
& \Leftrightarrow \quad \frac{|3 x+4 y|}{10} \leq \frac{9}{10} \max \left\{|x-y|, \frac{\frac{7|x|}{10} \cdot \frac{7|y|}{5}}{|x-y|}\right\} \\
& \Leftrightarrow \quad|3 x+4 y| \leq 9 \max \left\{|x-y|, \frac{49|x y|}{50|x-y|}\right\} .
\end{aligned}
$$

As a consequence, we have to prove that, for all distinct $x, y \in X$,

$$
\min \left\{\frac{7|x|}{20}, \frac{7|y|}{10}\right\} \leq|x-y| \quad \text { implies }|3 x+4 y| \leq 9 \max \left\{|x-y|, \frac{49|x y|}{50|x-y|}\right\} .
$$

We consider some cases and subcases.

Case (1) Assume that $x \leq 0 \leq y$. Then $d(x, y)=y-x$.

In this case,

$$
\begin{aligned}
& \frac{7|x|}{20} \leq|x-y| \quad \Leftrightarrow \quad-\frac{7 x}{20} \leq y-x \quad \Leftrightarrow \quad \frac{13 x}{20} \leq y, \quad \text { true because } x \leq 0 \leq y ; \\
& \frac{7|y|}{10} \leq|x-y| \quad \Leftrightarrow \quad \frac{7 y}{20} \leq y-x \quad \Leftrightarrow \quad x \leq \frac{13 y}{20}, \quad \text { true because } x \leq 0 \leq y .
\end{aligned}
$$

In any case, condition

$$
\min \left\{\frac{7|x|}{20}, \frac{7|y|}{10}\right\} \leq|x-y|
$$


holds. Since

$$
\begin{aligned}
& 3 x+4 y \leq 9(y-x) \quad \Leftrightarrow \quad 12 x \leq 5 y, \quad \text { true because } x \leq 0 \leq y \\
& -(3 x+4 y) \leq 9(y-x) \quad \Leftrightarrow \quad 6 x \leq 13 y, \quad \text { true because } x \leq 0 \leq y
\end{aligned}
$$

we conclude that

$$
|3 x+4 y| \leq 9|x-y|
$$

so (23) holds.

Case (2) Assume that $y \leq 0 \leq x$. Then $d(x, y)=x-y$.

In this case,

$$
\begin{aligned}
& \frac{7|x|}{20} \leq|x-y| \quad \Leftrightarrow \quad \frac{7 x}{20} \leq x-y \quad \Leftrightarrow \quad y \leq \frac{13 x}{20}, \quad \text { true because } y \leq 0 \leq x ; \\
& \frac{7|y|}{10} \leq|x-y| \quad \Leftrightarrow \quad-\frac{7 y}{20} \leq x-y \quad \Leftrightarrow \quad \frac{13 y}{20} \leq x, \quad \text { true because } y \leq 0 \leq x .
\end{aligned}
$$

In any case, condition

$$
\min \left\{\frac{7|x|}{20}, \frac{7|y|}{10}\right\} \leq|x-y|
$$

holds. Since

$$
\begin{aligned}
& 3 x+4 y \leq 9(x-y) \quad \Leftrightarrow \quad 13 y \leq 6 x, \quad \text { true because } y \leq 0 \leq x \\
& -(3 x+4 y) \leq 9(x-y) \quad \Leftrightarrow \quad 4 y \leq 12 x, \quad \text { true because } y \leq 0 \leq x
\end{aligned}
$$

we conclude that

$$
|3 x+4 y| \leq 9|x-y|
$$

so (23) holds.

Case (3) Assume that $0<x<y$. Hence $d(x, y)=y-x$ and $|3 x+4 y|=3 x+4 y$.

In this case,

$$
d(x, T x)=\frac{7 x}{20}<\frac{7 y}{20}<\frac{7 y}{10}=d(y, S y) .
$$

If

$$
d(T x, S y)=\frac{3 x+4 y}{10} \leq \frac{9}{10}|x-y|
$$

then (23) holds and this case is finished. On the contrary case, assume that

$$
\frac{3 x+4 y}{10}>\frac{9}{10}|x-y|
$$

Therefore

$$
\frac{3 x+4 y}{10}>\frac{9}{10}|x-y| \quad \Leftrightarrow \quad 3 x+4 y>9(y-x) \quad \Leftrightarrow \quad 12 x>5 y \quad \Leftrightarrow \quad \frac{5}{12} y<x .
$$

Then we know that:

$$
0<\frac{5}{12} y<x
$$

In particular, multiplying by $x$ and $y$,

$$
\frac{5}{12} y^{2}<x y \text { and } \frac{5}{12} x y<x^{2}
$$


In this case,

$$
\begin{aligned}
d(T x, S y) & \leq \frac{9}{10} \frac{d(x, T x) d(y, S y)}{d(x, y)} \Leftrightarrow \frac{3 x+4 y}{10} \leq \frac{9}{10} \frac{\frac{7 x}{10} \cdot \frac{7 y}{5}}{|x-y|} \\
& \Leftrightarrow \quad(3 x+4 y)(y-x) \leq \frac{441}{50} x y \quad \Leftrightarrow \quad 4 y^{2}-3 x^{2}-x y \leq \frac{441}{50} x y \\
& \Leftrightarrow \quad 200 y^{2}-150 x^{2}-50 x y \leq 441 x y \quad \Leftrightarrow \quad 200 y^{2}-150 x^{2} \leq 491 x y .
\end{aligned}
$$

To prove that this inequality holds, we use (24). Then:

$$
\begin{aligned}
& \frac{5}{12} y^{2}<x y \quad \Leftrightarrow \quad y^{2}<\frac{12}{5} x y \quad \Leftrightarrow \quad 200 y^{2}<200 \frac{12}{5} x y=480 x y ; \\
& \frac{5}{12} x y<x^{2} \quad \Leftrightarrow \quad-x^{2}<-\frac{5}{12} x y \quad \Leftrightarrow \quad-150 x^{2}<-150 \frac{5}{12} x y=-\frac{125}{2} x y .
\end{aligned}
$$

Adding the last two inequalities,

$$
200 y^{2}-150 x^{2} \leq 480 x y-\frac{125}{2} x y=\frac{835}{2} x y=417.5 x y<491 x y,
$$

so (25) guarantees that, in this case,

$$
d(T x, S y) \leq \frac{9}{10} \frac{d(x, T x) d(y, S y)}{d(x, y)} \leq \frac{9}{10} m(x, y) .
$$

Case (4) Assume that $0<y<x$. In this case, $d(x, y)=x-y$ and $|3 x+4 y|=3 x+4 y$.

If the inequality

$$
d(T x, S y)=\frac{3 x+4 y}{10} \leq \frac{9}{10}|x-y|=\frac{9}{10}(x-y)
$$

holds, then the contractivity condition (22) is true. On the contrary case, assume that

$$
\frac{3 x+4 y}{10}>\frac{9}{10}(x-y),
$$

that is,

$$
\frac{3 x+4 y}{10}>\frac{9}{10}(x-y) \Leftrightarrow 3 x+4 y>9 x-9 y \quad \Leftrightarrow \quad 13 y>6 x \quad \Leftrightarrow \quad \frac{6}{13} x<y .
$$

Then we know that

$$
0<\frac{6}{13} x<y
$$

Multiplying by $x$ and $y$, we deduce that

$$
\frac{6}{13} x^{2}<x y \text { and } \frac{6}{13} x y<y^{2}
$$

The contractivity condition is equivalent to:

$$
\begin{aligned}
d(T x, S y) \leq \frac{9}{10} \frac{d(x, T x) d(y, S y)}{d(x, y)} \quad \Leftrightarrow \quad \frac{3 x+4 y}{10} \leq \frac{9}{10} \frac{7 x}{|x-y|} \cdot \frac{7 y}{5} \\
\quad \Leftrightarrow \quad(3 x+4 y)(x-y) \leq \frac{441}{50} x y \quad \Leftrightarrow \quad 3 x^{2}+x y-4 y^{2} \leq \frac{441}{50} x y \\
\quad \Leftrightarrow \quad 150 x^{2}+50 x y-200 y^{2} \leq 441 x y \quad \Leftrightarrow \quad 150 x^{2}-200 y^{2} \leq 391 x y .
\end{aligned}
$$


To prove that this inequality holds, we use (26). Then:

$$
\begin{aligned}
\frac{6}{13} x^{2}<x y & \Leftrightarrow \quad x^{2}<\frac{13}{6} x y \quad \Leftrightarrow \quad 150 x^{2}<150 \frac{13}{6} x y=325 x y \\
\frac{6}{13} x y<y^{2} & \Leftrightarrow \quad-y^{2}<-\frac{6}{13} x y \quad \Leftrightarrow \quad-200 x^{2}<-200 \frac{6}{13} x y=-\frac{1200}{13} x y .
\end{aligned}
$$

Adding the last two inequalities,

$$
150 x^{2}-200 y^{2}<325 x y-\frac{1200}{13} x y=\frac{3025}{13} x y<233 x y<391 x y,
$$

so (27) guarantees that, in this case,

$$
d(T x, S y) \leq \frac{9}{10} \frac{d(x, T x) d(y, S y)}{d(x, y)} \leq \frac{9}{10} m(x, y)
$$

Case (5) Assume that $x<y<0$. It is sufficient to apply case 4 to $x^{\prime}=-x>0$ and $y^{\prime}=-y>0$ because $0<y^{\prime}<x^{\prime}, d\left(x^{\prime}, T x^{\prime}\right)=d(x, T x), d\left(y^{\prime}, S^{\prime}\right)=d(y, S y), d\left(x^{\prime}, y^{\prime}\right)=d(x, y)$ and so on.

Case (6) Assume that $y<x<0$. It is sufficient to apply case 3 to $x^{\prime}=-x>0$ and $y^{\prime}=-y>0$ because $0<x^{\prime}<y^{\prime}$.

In the following result, we replace the continuity of $T$ and $S$ by a distinct contractivity condition.

Theorem 3. Let $(X, d)$ be a complete metric space and let $T, S: X \rightarrow X$ be two mappings such that, for all $x, y \in X$,

$$
\frac{1}{2} \min \{d(x, T x), d(y, S y)\} \leq d(x, y) \quad \text { implies } \quad \eta(d(T x, S y), r(x, y)) \geq 0,
$$

where $\eta \in Z_{\Psi}$ and

$$
r(x, y)=\max \left\{d(x, y), \frac{[1+d(x, T x)] d(y, S y)}{1+d(x, y)}\right\} .
$$

Then $T$ and $S$ have a unique common fixed point.

Proof. Let $\psi \in Z_{\Psi}$ be a function associated to $\eta$ as in Definition 2. First of all, we prove that, under the contractivity condition, any fixed point of $T$ is a common fixed point of $S$ and $T$ (that is, if $x \in X$ is such that $T x=x$, then $S x=x$ ). To prove it, suppose that $x \in X$ is such that $T x=x$ and, by contradiction, assume that $S x \neq x$. Then

$$
r(x, x)=\max \left\{d(x, x), \frac{[1+d(x, T x)] d(x, S x)}{1+d(x, x)}\right\}=d(x, S x)>0 .
$$

Using $y=x$ in (28), since

$$
\begin{aligned}
\frac{1}{2} \min \{d(x, T x), d(x, S x)\} & =\frac{1}{2} \min \{0, d(x, S x)\} \\
& =0=d(x, x),
\end{aligned}
$$

then

$$
\eta(d(T x, S x), r(x, x)) \geq 0 .
$$

Since $d(x, S x)>0$, this means that

$$
\begin{aligned}
0 & \leq \eta(d(T x, S x), r(x, x))=\eta(d(x, S x), d(x, S x))< \\
& <\psi(d(x, S x))-\psi(d(x, S x))=0
\end{aligned}
$$


which is impossible. This proves the claim. In fact, swaping $T$ and $S$, we have checked that any fixed point of $T$ or $S$ is a common fixed point of $T$ and $S$.

Next, by starting from an arbitrary point $x_{0}$, we shall built up a recursive sequence $\left\{x_{n}\right\}$ defined as:

$$
x_{2 n+1}=T x_{2 n} \text {, and } x_{2 n+2}=S x_{2 n+1} \text { for each } n \in \mathbb{N} .
$$

If there is $n_{0} \in \mathbb{N}$ such that $x_{n_{0}}=x_{n_{0}+1}$, then $x_{n_{0}}$ is a fixed point of $T$ or $S$, so $x_{n_{0}}$ is a common fixed point of $T$ and $S$. In this case, the existence of some common fixed point of $T$ and $S$ is guaranteed. Through the rest of the proof, we suppose that

$$
x_{n} \neq x_{n+1} \quad \text { for all } n \in \mathbb{N} .
$$

In particular,

$$
r\left(x_{n}, x_{n+1}\right) \geq d\left(x_{n}, x_{n+1}\right)>0 \text { for all } n \in \mathbb{N} .
$$

We shall consider the following set in that will be useful in later stages of the proof.

$$
\mathcal{A}:=\left\{(x, y) \in X \times X: \frac{1}{2} \min \{d(x, T x), d(y, S y)\} \leq d(x, y)\right\} .
$$

Notice that $\mathcal{A}$ is non-empty because $\left(x_{2 n}, T x_{2 n}\right)=\left(x_{2 n}, x_{2 n+1}\right) \in \mathcal{A}$ for all $n \in \mathbb{N}$.

We divide the rest of the proof into five steps.

Step (1): We claim that

$$
d\left(x_{k+1}, x_{k+2}\right) \leq r\left(x_{k}, x_{k+1}\right)=d\left(x_{k}, x_{k+1}\right) \quad \text { for all } k \in \mathbb{N} .
$$

To see this, suppose that $k=2 n$ for some $n \in \mathbb{N}$. We have

$$
\begin{aligned}
\frac{1}{2} \min \left\{d\left(x_{2 n}, T x_{2 n}\right), d\left(x_{2 n+1}, S x_{2 n+1}\right)\right\} & =\frac{1}{2} \min \left\{d\left(x_{2 n}, x_{2 n+1}\right), d\left(x_{2 n+1}, x_{2 n+2}\right)\right\} \\
& \leq d\left(x_{2 n}, x_{2 n+1}\right) .
\end{aligned}
$$

So from $(28)$ and $\left(\eta_{1}\right)$ we have:

$$
\begin{aligned}
0 & \leq \eta\left(d\left(T x_{2 n}, S x_{2 n+1}\right), r\left(x_{2 n}, x_{2 n+1}\right)\right)=\eta\left(d\left(x_{2 n+1}, x_{2 n+2}\right), r\left(x_{2 n}, x_{2 n+1}\right)\right) \\
& <\psi\left(r\left(x_{2 n}, x_{2 n+1}\right)\right)-\psi\left(d\left(x_{2 n+1}, x_{2 n+2}\right)\right),
\end{aligned}
$$

and as $\psi$ is increasing, then

$$
d\left(x_{2 n+1}, x_{2 n+2}\right)<r\left(x_{2 n}, x_{2 n+1}\right) .
$$

On the other hand,

$$
\begin{aligned}
r\left(x_{2 n}, x_{2 n+1}\right) & =\max \left\{d\left(x_{2 n}, x_{2 n+1}\right), \frac{\left[1+d\left(x_{2 n}, T x_{2 n}\right)\right] d\left(x_{2 n+1}, S x_{2 n+1}\right)}{1+d\left(x_{2 n}, x_{2 n+1}\right)}\right\} \\
& =\max \left\{d\left(x_{2 n}, x_{2 n+1}\right), \frac{\left[1+d\left(x_{2 n}, x_{2 n+1}\right)\right] d\left(x_{2 n+1}, x_{2 n+2}\right)}{1+d\left(x_{2 n}, x_{2 n+1}\right)}\right\} \\
& =\max \left\{d\left(x_{2 n}, x_{2 n+1}\right), d\left(x_{2 n+1}, x_{2 n+2}\right)\right\} .
\end{aligned}
$$

So, if there exists some $n_{0} \in \mathbb{N}$ such that $d\left(x_{2 n_{0}+1}, x_{2 n_{0}+2}\right) \geq d\left(x_{2 n_{0}}, x_{2 n_{0}+1}\right)$, then

$$
r\left(x_{2 n_{0}}, x_{2 n_{0}+1}\right)=d\left(x_{2 n_{0}+1}, x_{2 n_{0}+2}\right)
$$


which contradicts (33). Hence, for each $n \in \mathbb{N}$,

$$
d\left(x_{2 n+1}, x_{2 n+2}\right)<d\left(x_{2 n}, x_{2 n+1}\right)
$$

and also

$$
r\left(x_{2 n}, x_{2 n+1}\right)=d\left(x_{2 n}, x_{2 n+1}\right) .
$$

Consequently, (32) is proved when $k \geq 0$ is an even number. By the same argument, one can verify that (32) holds when $k$ is an odd number.

Step (2): We prove that $\lim _{k \rightarrow \infty} d\left(x_{k}, x_{k+1}\right)=0$.

Since the sequence $\left\{d\left(x_{n}, x_{n+1}\right)\right\}_{n \geq 1}$ is non-increasing and bounded below, it is convergent to a real number $\gamma \geq 0$. Hence

$$
\lim _{n \rightarrow \infty} d\left(x_{n}, x_{n+1}\right)=\lim _{n \rightarrow \infty} r\left(x_{n}, x_{n+1}\right)=\gamma .
$$

We claim that $\gamma=0$. To prove the claim, let define

$$
\Omega=\{(d(T x, S y), r(x, y)):(x, y) \in \mathcal{A}\} .
$$

By (28) and definition of $\mathcal{A}$, it follows that

$$
\eta(t, s) \geq 0 \quad \text { for all }(t, s) \in \Omega \backslash\{(0,0)\} .
$$

For each $n \geq 0$ we have

$$
\begin{aligned}
\frac{1}{2} \min \left\{d\left(x_{2 n}, T x_{2 n}\right), d\left(x_{2 n+1}, S x_{2 n+1}\right)\right\} & =\frac{1}{2} \min \left\{d\left(x_{2 n}, x_{2 n+1}\right), d\left(x_{2 n+1}, x_{2 n+2}\right)\right\} \\
& \leq d\left(x_{2 n}, x_{2 n+1}\right) .
\end{aligned}
$$

Thus $\left(x_{2 n}, x_{2 n+1}\right) \in \mathcal{A}$ for each $n \geq 0$. Consequently, (35) implies that

$$
\eta\left(d\left(T x_{2 n}, S x_{2 n+1}\right), r\left(x_{2 n}, x_{2 n+1}\right)\right) \geq 0 \quad \text { for all } n \in \mathbb{N} .
$$

So

$$
\limsup _{n \rightarrow \infty} \eta\left(d\left(x_{2 n+1}, x_{2 n+2}\right), r\left(x_{2 n}, x_{2 n+1}\right)\right) \geq 0 .
$$

Next, reasoning by contradiction, suppose that $\gamma>0$. From (34) we have

$$
\left.\lim _{n \rightarrow \infty} d\left(x_{2 n+1}, x_{2 n+2}\right)=\lim _{n \rightarrow \infty} r\left(x_{2 n}, x_{2 n+1}\right)\right)=\gamma>0 .
$$

Therefore, from $\left(\eta_{2}\right)$,

$$
\limsup _{n \rightarrow \infty} \eta\left(d\left(x_{2 n+1}, x_{2 n+2}\right), r\left(x_{2 n}, x_{2 n+1}\right)\right)<0,
$$

which contradicts (36). So the claim is proved and we obtain that

$$
\lim _{n \rightarrow \infty} d\left(x_{n}, x_{n+1}\right)=\lim _{n \rightarrow \infty} r\left(x_{n}, x_{n+1}\right)=0 .
$$

Step (3): We claim that $\left\{x_{n}\right\}$ is a Cauchy sequence. To show that $\left\{x_{n}\right\}$ is a Cauchy sequence, because of (37), it is enough to show that the subsequence $\left\{x_{2 n}\right\}$ is a Cauchy sequence. On contrary, suppose that $\left\{x_{2 n}\right\}$ is not a Cauchy sequence. By Lemma 1 there exist $\varepsilon_{0}>0$ and subsequences $\left\{x_{2 m_{k}}\right\}$ 
and $\left\{x_{2 n_{k}}\right\}$ of $\left\{x_{n}\right\}$ such that $n_{k}$ is the smallest index for which $n_{k}>m_{k}>k$ and $d\left(x_{2 m_{k}}, x_{2 n_{k}}\right) \geq \varepsilon_{0}$ and

(li) $\lim _{k \rightarrow \infty} d\left(x_{2 m_{k}}, x_{2 n_{k}}\right)=\varepsilon_{0}$.

(l2) $\lim _{k \rightarrow \infty} d\left(x_{2 m_{k}-1}, x_{2 n_{k}}\right)=\varepsilon_{0}$.

(l) $\lim _{k \rightarrow \infty} d\left(x_{2 m_{k}}, x_{2 n_{k}+1}\right)=\varepsilon_{0}$.

$\left(l_{4}\right) \lim _{k \rightarrow \infty} d\left(x_{2 m_{k}-1}, x_{2 n_{k}+1}\right)=\varepsilon_{0}$.

Therefore, from the definition of $r(x, y)$ we have

$$
\begin{aligned}
\lim _{k \rightarrow \infty} r\left(x_{2 n_{k}}, x_{2 m_{k}-1}\right) & =\lim _{k \rightarrow \infty} \max \left\{d\left(x_{2 n_{k}}, x_{2 m_{k}-1}\right), \frac{\left[1+d\left(x_{2 n_{k}}, T x_{2 n_{k}}\right)\right] d\left(x_{2 m_{k}-1}, S x_{2 m_{k}-1}\right)}{1+d\left(x_{2 n_{k}}, x_{2 m_{k}-1}\right)}\right\} \\
& =\max \left\{\varepsilon_{0}, 0\right\}=\varepsilon_{0} .
\end{aligned}
$$

So

$$
\lim _{k \rightarrow \infty} d\left(x_{2 m_{k}}, x_{2 n_{k}+1}\right)=\lim _{k \rightarrow \infty} r\left(x_{2 m_{k}-1}, x_{2 n_{k}}\right)=\varepsilon_{0}>0 .
$$

Hence, $\left(\eta_{2}\right)$ implies that

$$
\limsup _{n \rightarrow \infty} \eta\left(d\left(x_{2 m_{k}}, x_{2 n_{k}+1}\right), r\left(x_{2 m_{k}-1}, x_{2 n_{k}}\right)\right)<0 .
$$

On the other hand, we claim that for sufficiently large $k \in \mathbb{N}$, if $n_{k}>m_{k}>k$, then

$$
\frac{1}{2} \min \left\{d\left(x_{2 n_{k}}, T x_{2 n_{k}}\right), d\left(x_{2 m_{k}-1}, S x_{2 m_{k}-1}\right)\right\} \leq d\left(x_{2 n_{k}}, x_{2 m_{k}-1}\right) .
$$

Indeed, since $n_{k}>m_{k}$ and $\left\{d\left(x_{n}, x_{n+1}\right)\right\}$ is non-increasing we have

$$
\begin{aligned}
d\left(x_{2 n_{k}}, T x_{2 n_{k}}\right) & =d\left(x_{2 n_{k}}, x_{2 n_{k}+1}\right) \leq d\left(x_{2 m_{k}+1}, x_{2 m_{k}}\right) \leq d\left(x_{2 m_{k}}, x_{2 m_{k}-1}\right) \\
& =d\left(x_{2 m_{k}-1}, S x_{2 m_{k}-1}\right) .
\end{aligned}
$$

So, the left hand side of inequality (39) is equal to

$$
\frac{1}{2} d\left(x_{2 n_{k}}, T x_{2 n_{k}}\right)=\frac{1}{2} d\left(x_{2 n_{k}}, x_{2 n_{k}+1}\right)
$$

Therefore, we must show that, for sufficiently large $k \in \mathbb{N}$, if $n_{k}>m_{k}>k$, then

$$
d\left(x_{2 n_{k}}, x_{2 n_{k}+1}\right) \leq d\left(x_{2 n_{k}}, x_{2 m_{k}-1}\right) .
$$

According to (37), there exists $k_{1} \in \mathbb{N}$ such that for any $k>k_{1}$,

$$
d\left(x_{2 n_{k}}, x_{2 n_{k}+1}\right)<\frac{\varepsilon_{0}}{2}
$$

There exists $k_{2} \in \mathbb{N}$ such that for any $k>k_{2}$,

$$
d\left(x_{2 m_{k}-1}, x_{2 m_{k}}\right)<\frac{\varepsilon_{0}}{2}
$$


Hence, for any $k>\max \left\{k_{1}, k_{2}\right\}$ and $n_{k}>m_{k}>k$, we have

$$
\begin{aligned}
\varepsilon_{0} & \leq d\left(x_{2 n_{k}}, x_{2 m_{k}}\right) \leq d\left(x_{2 n_{k}}, x_{2 m_{k}-1}\right) d\left(x_{2 m_{k}-1}, x_{2 m_{k}}\right) \\
& \leq d\left(x_{2 n_{k}}, x_{2 m_{k}-1}\right)+\frac{\varepsilon_{0}}{2} .
\end{aligned}
$$

So, one concludes that

$$
\frac{\varepsilon_{0}}{2} \leq d\left(x_{2 n_{k}}, x_{2 m_{k}-1}\right) .
$$

Thus we obtain that for any $k>\max \left\{k_{1}, k_{2}\right\}$ and $n_{k}>m_{k}>k$,

$$
d\left(x_{2 n_{k}}, x_{2 n_{k}+1}\right) \leq \frac{\varepsilon_{0}}{2} \leq d\left(x_{2 n_{k}}, x_{2 m_{k}-1}\right) .
$$

So (39) is proved. Therefore, by (28) and the definition of $\mathcal{A}$, for sufficiently large $k \in \mathbb{N}$, if $n_{k}>m_{k}>k$, then $\left(x_{2 n_{k}}, x_{2 m_{k}-1}\right) \in \mathcal{A}$. Consequently, for sufficiently large $k \in \mathbb{N}$, if $n_{k}>m_{k}>k$ then

$$
\eta\left(d\left(T x_{2 n_{k}}, S x_{2 m_{k}-1}\right), r\left(x_{2 n_{k}}, x_{2 m_{k}-1}\right)\right) \geq 0 .
$$

So

$$
\limsup _{k \rightarrow \infty} \eta\left(d\left(x_{2 n_{k}+1}, x_{2 m_{k}}\right), r\left(x_{2 n_{k}}, x_{2 m_{k}-1}\right)\right) \geq 0,
$$

which contradicts (38). This contradiction demonstrates that $\left\{x_{n}\right\}$ is a Cauchy sequence and, since $X$ is complete, there exists $u \in X$ such that $\left\{x_{n}\right\} \rightarrow u$ as $n \rightarrow \infty$.

Step (4): $u$ is a common fixed point of $T$ and $S$.

If $u$ is a fixed point of $T$ or $S$, we have demonstrated at the beginning of the proof that $u$ is a common fixed point of $T$ and $S$, and this step is finished. Next, suppose that $d(u, T u)>0$ and $d(u, S u)>0$, and we will get a contradiction.

If there are infinitely many positive even integers $\left\{2 n_{k}\right\}$ such that $x_{2 n_{k}}=u$ for all $k \in \mathbb{N}$, then $x_{2 n_{k}+1}=T x_{2 n_{k}}=T u$ for all $k \in \mathbb{N}$, and as $\left\{x_{2 n_{k}+1}\right\} \rightarrow u$, then $T u=u$, which is false. The same is true if there are infinitely many positive odd integers $\left\{2 n_{k}+1\right\}$ such that $x_{2 n_{k}+1}=u$ for all $k \in \mathbb{N}$, because $x_{2 n_{k}+2}=T x_{2 n_{k}+1}=S u$ for all $k \in \mathbb{N}$. In general, if there is a subsequence $\left\{x_{n_{k}}\right\}$ such that $x_{n_{k}}=u$ for all $k \in \mathbb{N}$, then we can guarantee that $u$ is a common fixed point of $T$ and $S$. On the contrary case, without loss of generality, suppose that

$$
d\left(x_{n}, u\right) \neq 0 \text { for each } n \geq 0 .
$$

Since $\left\{d\left(x_{2 n}, u\right)\right\} \rightarrow 0$ and $\left\{d\left(x_{2 n}, x_{2 n+1}\right)\right\} \rightarrow 0$, then

$$
\begin{aligned}
\lim _{n \rightarrow \infty} r\left(u, x_{2 n}\right) & =\lim _{n \rightarrow \infty} \max \left\{d\left(x_{2 n}, u\right), \frac{\left[1+d\left(x_{2 n}, T x_{2 n}\right)\right] d(u, S u)}{1+d\left(x_{2 n}, u\right)}\right\} \\
& =\lim _{n \rightarrow \infty} \max \left\{d\left(x_{2 n}, u\right), \frac{\left[1+d\left(x_{2 n}, x_{2 n+1}\right)\right] d(u, S u)}{1+d\left(x_{2 n}, u\right)}\right\} \\
& =\max \{0, d(u, S u)\}=d(u, S u)>0 .
\end{aligned}
$$

This proves that

$$
\lim _{n \rightarrow \infty} r\left(u, x_{2 n}\right)=d(u, S u) .
$$

In the same way, one can show that

$$
\lim _{n \rightarrow \infty} r\left(u, x_{2 n+1}\right)=d(T u, u) .
$$


Now, we claim that for each $n \geq 0$, at least one of the following inequalities is true:

$$
\frac{1}{2} d\left(x_{2 n}, x_{2 n+1}\right) \leq d\left(x_{2 n}, u\right)
$$

or

$$
\frac{1}{2} d\left(x_{2 n+1}, x_{2 n+2}\right) \leq d\left(x_{2 n}, u\right) .
$$

If these inequalities are both false for some $n_{0} \geq 0$, then we get

$$
\begin{aligned}
d\left(x_{2 n_{0}}, x_{2 n_{0}+1}\right) & \leq d\left(x_{2 n_{0}}, u\right)+d\left(u, x_{2 n_{0}+1}\right)<\frac{1}{2}\left[d\left(x_{2 n_{0}}, x_{2 n_{0}+1}\right)+d\left(x_{2 n_{0}+1}, x_{2 n_{0}+2}\right)\right] \\
& \leq \frac{1}{2}\left[d\left(x_{2 n_{0}}, x_{2 n_{0}+1}\right)+d\left(x_{2 n_{0}}, x_{2 n_{0}+1}\right)\right]=d\left(x_{2 n_{0}}, x_{2 n_{0}+1}\right)
\end{aligned}
$$

which is a contradiction, and the claim is proved. So, one can consider the following two subcases.

Subcase (4.1): The inequality (42) holds for infinitely many $n \geq 0$. In this case, for infinitely many $n \geq 0$ we have

$$
\begin{aligned}
\frac{1}{2} \min \left\{d\left(x_{2 n}, T x_{2 n}\right), d(u, S u)\right\} & =\frac{1}{2} \min \left\{d\left(x_{2 n}, x_{2 n+1}\right), d(u, S u)\right\} \\
& \leq \frac{1}{2} d\left(x_{2 n}, x_{2 n+1}\right) \leq d\left(x_{2 n}, u\right) .
\end{aligned}
$$

Therefore $\left(x_{2 n}, u\right) \in \mathcal{A}$. Thus

$$
\eta\left(d\left(T x_{2 n}, S u\right), r\left(x_{2 n}, u\right)\right) \in \Omega \backslash\{(0,0)\} .
$$

Consequently, from (35) it is seen that for infinitely many $n \geq 0$,

$$
\eta\left(d\left(T x_{2 n}, S u\right), r\left(x_{2 n}, u\right)\right) \geq 0 .
$$

Therefore, for infinitely many $\left\{n_{k}\right\}_{k \geq 0}$,

$$
\limsup _{k \rightarrow \infty} \eta\left(d\left(x_{2 n_{k}+1}, S u\right), r\left(x_{2 n_{k}}, u\right)\right) \geq 0 .
$$

However,

$$
\lim _{k \rightarrow \infty} d\left(x_{2 n_{k}+1}, S u\right)=\lim _{k \rightarrow \infty} r\left(x_{2 n_{k}}, u\right)=d(u, S u)>0,
$$

and from $\left(\eta_{2}\right)$ we have

$$
\limsup _{k \rightarrow \infty} \eta\left(d\left(x_{2 n_{k}+1}, S u\right), r\left(x_{2 n_{k}}, u\right)\right)<0,
$$

which contradicts (44).

Subcase (4.2): The inequality (42) only holds for finitely many $n \geq 0$.

In this case, there exists $n_{0} \geq 0$ such that (43) is true for any $n \geq n_{0}$. Similar to subcase (4.1), one can prove that (43) also leads to a contradiction unless $u$ is a fixed point of $T$ or $S$.

As a consequence, in any case, $u$ is a common fixed point of $T$ and $S$.

Step (5): The common fixed point of $T$ and $S$ is unique.

Suppose that $u$ and $v$ are two distinct common fixed points of $T$ and $S$, that is, $T u=S u=u$ and $T v=S v=v$. Hence $d(u, v)>0$ and

$$
r(u, v)=\max \left\{d(u, v), \frac{[1+d(u, T u)] d(v, S v)}{1+d(u, v)}\right\}=d(u, v)>0 .
$$


Since

$$
\frac{1}{2} \min \{d(u, T u), d(v, S v)\}=0<d(u, v)
$$

we deduce from (28) that

$$
\begin{aligned}
0 & \leq \eta(d(T u, S v), r(u, v))=\eta(d(u, v), d(u, v)) \\
& <\psi(d(u, v))-\psi(d(u, v))=0,
\end{aligned}
$$

which is a contradiction. Hence, $T$ and $S$ have a unique common fixed point.

\section{Consequences}

In this section, we illustrate the applicability of the previous theorems by showing they cover a lot of distinct cases which permit us to deduce several results under different hypotheses and contractivity conditions. For instance, the following corollary is an immediate consequence of Theorem 2 by removing the restriction

$$
\frac{1}{2} \min \{d(x, T x), d(y, S y)\} \leq d(x, y) .
$$

Corollary 1. Let $(X, d)$ be a complete metric space and $T$ and $S$ be two continuous self-maps on $X$ such that, for all $x, y \in X$,

$$
\eta(d(T x, S y), m(x, y)) \geq 0
$$

where $\eta \in Z_{\Psi}$ and

$$
m(x, y)= \begin{cases}d(T x, S x), & \text { if } x=y, \\ \max \left\{d(x, y), \frac{d(x, T x) d(y, S y)}{d(x, y)}\right\}, & \text { if } x \neq y .\end{cases}
$$

Then $T$ and $S$ have a unique common fixed point.

Corollary 2. Let $(X, d)$ be a complete metric space and let $T$ be continuous self-map on $X$. If there exists $\eta \in Z_{\Psi}$ such that, for all $x, y \in X$,

$$
\frac{1}{2} d(x, T x) \leq d(x, y) \quad \text { implies } \quad \eta\left(d(T x, T y), m_{T}(x, y)\right) \geq 0,
$$

where

$$
m_{T}(x, y)= \begin{cases}0, & \text { if } x=y, \\ \max \left\{d(x, y), \frac{d(x, T x) d(y, T y)}{d(x, y)}\right\}, & \text { if } x \neq y\end{cases}
$$

then T has a unique fixed point.

Proof. Choose $S=T$ in Theorem 2 and we get the proof.

The following corollary is a consequence of Corollary 2.

Corollary 3. Let $(X, d)$ be a complete metric space and let $T$ be a continuous self-map on $X$. If there exists $\eta \in Z_{\Psi}$ such that, for all $x, y \in X$,

$$
\eta\left(d(T x, T y), m_{T}(x, y)\right) \geq 0
$$

where

$$
m_{T}(x, y)= \begin{cases}0, & \text { if } x=y, \\ \max \left\{d(x, y), \frac{d(x, T x) d(y, T y)}{d(x, y)}\right\}, & \text { if } x \neq y\end{cases}
$$

then $\mathrm{T}$ has a unique fixed point. 
Corollary 4. Let $(X, d)$ be a complete metric space and let $T$ and $S$ be two continuous self-maps on $X$ such that, for every $x, y \in X$,

$$
\begin{aligned}
\frac{1}{2} \min \{d(x, T x), d(y, S y)\} \leq d(x, y) \quad \text { implies } \\
\psi(d(T x, S y)) \leq \psi(m(x, y))-\phi(m(x, y))
\end{aligned}
$$

where $\psi:[0,+\infty) \rightarrow[0,+\infty)$ is continuous nondecreasing function such that $\psi(t)=0$ if, and only if, $t=0$,

$$
m(x, y)= \begin{cases}d(T x, S x), & \text { if } x=y \\ \max \left\{d(x, y), \frac{d(x, T x) d(y, S y)}{d(x, y)}\right\}, & \text { if } x \neq y\end{cases}
$$

and $\phi:[0,+\infty) \rightarrow[0,+\infty)$ is a function such that $\liminf _{s \rightarrow t} \phi(s)>0$ for each $t>0$, and $\phi(t)=0$ if, and only if, $t=0$. Then $T$ and $S$ have a unique common fixed point.

Proof. Define $\eta(t, s)=\psi(s)-\phi(s)-\psi(t)$ for all $t, s \geq 0$. Then $\eta$ is a $\Psi$-simulation function. Indeed, $\psi \in \Psi$ and $\left(\eta_{1}\right)$ clearly holds. On the other hand, if $\left\{t_{n}\right\},\left\{s_{n}\right\}$ are two sequences in $(0,+\infty)$ such that

$$
\lim _{n \rightarrow \infty} t_{n}=\lim _{n \rightarrow \infty} s_{n}>0
$$

then, we have

$$
\limsup _{n \rightarrow \infty} \eta\left(t_{n}, s_{n}\right)=\limsup _{n \rightarrow \infty}\left(\psi\left(s_{n}\right)-\phi\left(s_{n}\right)-\psi\left(t_{n}\right)\right) \leq 0-\liminf _{n \rightarrow \infty} \phi\left(s_{n}\right)<0 .
$$

So $\left(\eta_{2}\right)$ holds and we can apply Theorem 2 to complete the proof.

Remark 2. In some of the following results we will consider two functions $\psi, \phi:[0,+\infty) \rightarrow[0,+\infty)$ such that:

(i) $\psi$ is a continuous non-decreasing function and $\psi(t)=0$ if, and only if, $t=0$.

(ii) $\phi$ is lower semi-continuous with $\phi(t)=0$ if, and only if, $t=0$.

Corollary 5. Let $(X, d)$ be a complete metric space and let $T$ and $S$ be two continuous self-maps on $X$ such that, for every $x, y \in X$,

$$
\frac{1}{2} \min \{d(x, T x), d(y, S y)\} \leq d(x, y) \quad \text { implies } \quad \psi(d(T x, S y)) \leq \psi(m(x, y))-\phi(m(x, y)),
$$

where $\psi$ and $\phi$ are given as in Remark 2 and

$$
m(x, y)= \begin{cases}d(T x, S x), & \text { if } x=y \\ \max \left\{d(x, y), \frac{d(x, T x) d(y, S y)}{d(x, y)}\right\}, & \text { if } x \neq y\end{cases}
$$

Then $T$ and $S$ have a unique common fixed point.

Proof. Since $\phi$ is lower semi-continuous, if

$$
\lim _{n \rightarrow \infty} s_{n}=\ell>0
$$

then

$$
\liminf _{n \rightarrow \infty} \phi\left(s_{n}\right) \geq \phi(\ell)>0 .
$$

So one can apply Corollary 4. 
Corollary 6. Let $(X, d)$ be a complete metric space and let $T$ and $S$ be two continuous self-maps on $X$ such that, for every $x, y \in X$,

$$
\begin{array}{r}
\frac{1}{2} \min \{d(x, T x), d(y, S y)\} \leq d(x, y) \quad \text { implies } \\
\psi(d(T x, S y)) \leq \rho(m(x, y)) \psi(m(x, y))
\end{array}
$$

where $\psi \in \Psi, m(x, y)$ is defined as in Corollary 5 and $\rho:[0,+\infty) \rightarrow[0,1)$ is a function such that $\rho(t)=0$ if, and only if, $t=0$, and $\limsup _{t \rightarrow s} \rho(t)<1$ for each $s>0$. Then $T$ and $S$ have a unique common fixed point.

Proof. Take $\eta(t, s)=\rho(s) \psi(s)-\psi(t)$ for all $t, s \geq 0$. One can easily show that $\eta$ is a $\Psi$-simulation function. Now this consequence follows from Theorem 2.

Corollary 7. Let $(X, d)$ be a complete metric space and let $T$ and $S$ be two continuous maps on $X$ such that, for every $x, y \in X$,

$$
\begin{aligned}
\frac{1}{2} \min \{d(x, T x), d(y, S y)\} & \leq d(x, y) \quad \text { implies } \\
\psi((d(T x, S y))+\phi(d(T x, S y)) & \leq \psi(m(x, y))
\end{aligned}
$$

where $\psi \in \Psi, m(x, y)$ is defined as in Corollary 5 and $\phi:[0,+\infty) \rightarrow[0,+\infty)$ is a function such that $\liminf _{s \rightarrow t} \phi(s)>0$ for each $t>0$, and $\phi(t)=0$ if, and only if, $t=0$. Then $T$ and $S$ have a unique common fixed point.

Proof. Take $\eta(t, s)=\psi(s)-\phi(s)-\psi(t)$ for all $t, s \geq 0$. Then similar to the proof of Corollary 4 , one can see that $\eta$ is a $\Psi$-simulation function. So by applying Theorem 2 the proof is completed.

Corollary 8. Let $(X, d)$ be a complete metric space and let $T$ and $S$ be two continuous self-maps on $X$ such that, for every $x, y \in X$,

$$
\begin{gathered}
\frac{1}{2} \min \{d(x, T x), d(y, S y)\} \leq d(x, y) \quad \text { implies } \\
\psi((d(T x, S y)) \leq \phi(\psi(m(x, y)))
\end{gathered}
$$

where $\psi \in \Psi, m(x, y)$ is defined as in Corollary 5 and $\phi:[0,+\infty) \rightarrow[0,+\infty)$ is a function such that for each $t>0, \phi(t)<t$ and $\lim \sup \phi(s)<t$ and $\phi(t)=0$ if, and only if, $t=0$. Then $T$ and $S$ have a unique common fixed point.

Proof. Define $\eta(t, s)=\phi(\psi(s))-\psi(t)$ for all $t, s \geq 0$. Then $\left(\eta_{1}\right)$ holds. On the other hand, if $\left\{t_{n}\right\},\left\{s_{n}\right\}$ are two sequences in $(0,+\infty)$ such that

$$
\lim _{n \rightarrow \infty} s_{n}=\ell>0
$$

then, the continuity of $\psi$ and the properties of $\phi$ show that

$$
\limsup _{n \rightarrow \infty} \eta\left(t_{n}, s_{n}\right)=\limsup _{n \rightarrow \infty} \phi\left(\psi\left(s_{n}\right)\right)-\psi\left(t_{n}\right)<\psi(\ell)-\psi(\ell)=0
$$

Therefore $\left(\eta_{2}\right)$ holds and one can apply Theorem 2 to complete the proof. 
Remark 3. Suppose that $\psi \in \Psi$ and $\phi:[0,+\infty) \rightarrow[0,+\infty)$ is an upper semi-continuous function such that $\phi(t)<t$ for each $t>0$ and $\phi(t)=0$ if, and only if, $t=0$. Then for any sequence $\left\{s_{n}\right\}$ in $(0,+\infty)$ with $\lim _{n \rightarrow \infty} s_{n}=\ell>0$, one can obtain that

$$
\limsup _{n \rightarrow \infty} \phi\left(\psi\left(s_{n}\right)\right)<\psi(\ell)
$$

So, by applying the same argument as in Corollary 8, one can prove the following consequence.

Corollary 9. Let $(X, d)$ be a complete metric space and $T$ and $S$ be two continuous self-maps on $X$ such that for every $x, y \in X$,

$$
\begin{gathered}
\frac{1}{2} \min \{d(x, T x), d(y, S y)\} \leq d(x, y) \quad \text { implies } \\
\psi((d(T x, S y)) \leq \phi(\psi(m(x, y)))
\end{gathered}
$$

where $\psi \in \Psi, m(x, y)$ is defined as in Corollary 5 and $\phi:[0,+\infty) \rightarrow[0,+\infty)$ is an upper semi-continuous function such that $\phi(t)<t$ for each $t>0$, and $\phi(t)=0$ if, and only if, $t=0$. Then $T$ and $S$ have a unique common fixed point.

Definition 3. Let $(X, d)$ be a complete metric space, let $T: X \rightarrow X$ be a continuous mapping and let $\eta \in Z$. Then $T$ is called a generalized Z-contraction with respect to $\eta$ if the following condition is satisfied:

$$
\eta\left(d(T x, T y), m_{T}(x, y)\right) \geq 0
$$

for all $x, y \in X$, where

$$
m_{T}(x, y)= \begin{cases}0, & \text { if } x=y \\ \max \left\{d(x, y), \frac{d(x, T x) d(y, T y)}{d(x, y)}\right\}, & \text { if } x \neq y\end{cases}
$$

Theorem 4. Every generalized Z-contraction on a complete metric space has a unique fixed point.

Proof. It is an obvious consequence of Corollary 1 by letting $S=T$.

Theorem 5. Let $(X, d)$ be a complete metric space and let $T: X \rightarrow X$ be continuous mapping such that, for all $x, y \in X$,

$$
\frac{1}{2} d(x, T x) \leq d(x, y) \quad \text { implies } \quad \psi(d(T x, T y)) \leq \psi\left(m_{T}(x, y)\right)-\phi\left(m_{T}(x, y)\right),
$$

where $\psi$ and $\phi$ are given as in Remark 2 and

$$
m_{T}(x, y)= \begin{cases}0, & \text { if } x=y \\ \max \left\{d(x, y), \frac{d(x, T x) d(y, T y)}{d(x, y)}\right\}, & \text { if } x \neq y\end{cases}
$$

Then T has a unique fixed point.

Proof. It follows from Corollary 5 when $S=T$.

Theorem 6. Let $(X, d)$ be a complete metric space and let $T$ and $S$ be two continuous self-maps on $X$ such that, for every $x, y \in X$,

$$
\frac{1}{2} \min \{d(x, T x), d(y, S y)\} \leq d(x, y) \quad \text { implies } \quad \psi(d(T x, S y)) \leq \psi(m(x, y))-\phi(m(x, y))
$$


where $\psi$ and $\phi$ are given as in Theorem 5 and

$$
m(x, y)= \begin{cases}d(T x, S x), & \text { if } x=y, \\ \max \left\{d(x, y), \frac{d(x, T x) d(y, S y)}{d(x, y)}\right\}, & \text { if } x \neq y .\end{cases}
$$

Then $T$ and $S$ have a unique common fixed point.

Proof. Since $\phi$ is lower semi-continuous, if $\lim _{n \rightarrow+\infty} S_{n}=\ell>0$, then $\liminf _{n \rightarrow+\infty} \phi\left(S_{n}\right) \geq \phi(\ell)>0$. Hence, by applying Corollary 4 , we get the result.

The following example shows that Theorem 2 is a genuine generalization of the Corollary 1.

Example 6. Let $X=\{(0,0),(0,10),(10,0),(10,10),(10,12)\}$ be endowed with the metric d defined by

$$
d\left(\left(x_{1}, x_{2}\right),\left(y_{1}, y_{2}\right)\right)=\left|x_{1}-y_{1}\right|+\left|x_{2}-y_{2}\right| .
$$

Let $T$ and $S$ be the continuous self-mappings on $X$ defined as follows:

$$
T\left(x_{1}, x_{2}\right)=\left(\min \left\{x_{1}, x_{2}\right\}, 0\right),
$$

and

$$
S\left(x_{1}, x_{2}\right)=\left(0, \min \left\{x_{1}, x_{2}\right\}\right)
$$

for all $\left(x_{1}, x_{2}\right) \in X$. For any $\eta \in Z_{\Psi}$, the mappings $T$ and $S$ do not satisfy the condition (45) of Corollary 1 at $x_{0}=(10,12)$ and $y_{0}=(10,10)$. However, by choosing $\eta(t, s)=\frac{11}{100} s-t$ for all $t, s \in[0,+\infty)$, it is readily verified that $\eta$ is a $\Psi$-simulation function where $\psi$ is the identity function on $[0,+\infty)$, and all the hypothesis of Theorem 2 are verified.

The following corollary is a consequence of Theorem 3.

Corollary 10. Let $(X, d)$ be a complete metric space and let $T$ and $S$ be two self-maps on $X$ such that, for all $x, y \in X$,

$$
\eta(d(T x, S y), r(x, y)) \geq 0,
$$

where $\eta \in Z_{\Psi}$ and

$$
r(x, y)=\max \left\{d(x, y), \frac{[1+d(x, T x)] d(y, S y)}{1+d(x, y)}\right\} .
$$

Then $T$ and $S$ have a unique common fixed point.

Corollary 11. Let $(X, d)$ be a complete metric space and let $T$ be self-map on $X$. If there exists $\eta \in Z_{\Psi}$ such that, for all $x, y \in X$,

$$
\frac{1}{2} d(x, T x) \leq d(x, y) \quad \text { implies } \quad \eta(d(T x, T y), r(x, y)) \geq 0,
$$

where

$$
r_{T}(x, y)=\max \left\{d(x, y), \frac{[1+d(x, T x)] d(y, T y)}{1+d(x, y)}\right\},
$$

then T has a unique fixed point.

Proof. Putting $S=T$ in Theorem 3 we get the proof.

The following corollary is an application of Corollary 11. 
Corollary 12. Let $(X, d)$ be a complete metric space and let $T$ be self-map on $X$. If there exists $\eta \in Z_{\Psi}$ such that, for all $x, y \in X$,

$$
\eta\left(d(T x, T y), r_{T}(x, y)\right) \geq 0,
$$

where $r_{T}(x, y)$ is defined as in Corollary 11 , then $T$ has a unique fixed point.

Corollary 13. Let $(X, d)$ be a complete metric space and let $T$ and $S$ be two self-maps on $X$ such that, for every $x, y \in X$,

$$
\begin{gathered}
\frac{1}{2} \min \{d(x, T x), d(y, S y)\} \leq d(x, y) \quad \text { implies } \\
\psi(d(T x, S y)) \leq \psi(r(x, y))-\phi(r(x, y)),
\end{gathered}
$$

where $\psi:[0,+\infty) \rightarrow[0,+\infty)$ is a continuous nondecreasing function and $\psi(t)=0$ if, and only if, $t=0$,

$$
r(x, y)=\max \left\{d(x, y), \frac{[1+d(x, T x)] d(y, S y)}{1+d(x, y)}\right\},
$$

and $\phi:[0,+\infty) \rightarrow[0,+\infty)$ is a function such that $\liminf _{s \rightarrow t} \phi(s)>0$ for each $t>0$, and $\phi(t)=0$ if, and only if, $t=0$. Then $T$ and $S$ have a unique common fixed point.

Proof. Define $\eta(t, s)=\psi(s)-\phi(s)-\psi(t)$ for all $t, s \geq 0$. In the proof of Corollary 4 we showed that $\eta$ is a $\Psi$-simulation function. Hence, we can apply Theorem 3 to complete the proof.

Corollary 14. Let $(X, d)$ be a complete metric space and let $T$ and $S$ be two self-maps on $X$ such that, for every $x, y \in X$,

$$
\frac{1}{2} \min \{d(x, T x), d(y, S y)\} \leq d(x, y) \quad \text { implies } \quad \psi(d(T x, S y)) \leq \psi(r(x, y))-\phi(r(x, y)),
$$

where $\psi$ and $\phi$ are given as in Remark 8, and

$$
r(x, y)=\max \left\{d(x, y), \frac{[1+d(x, T x)] d(y, S y)}{1+d(x, y)}\right\} .
$$

Then $T$ and $S$ have a unique common fixed point.

Proof. Since $\phi$ is lower semi-continuous, if

$$
\lim _{n \rightarrow \infty} s_{n}=\ell>0
$$

then

$$
\liminf _{n \rightarrow \infty} \phi\left(s_{n}\right) \geq \phi(\ell)>0
$$

So, one can apply Corollary 13.

Corollary 15. Let $(X, d)$ be a complete metric space and let $T$ and $S$ be two self-maps on $X$ such that, for every $x, y \in X$,

$$
\begin{array}{r}
\frac{1}{2} \min \{d(x, T x), d(y, S y)\} \leq d(x, y) \quad \text { implies } \\
\psi(d(T x, S y)) \leq \rho(r(x, y)) \psi(r(x, y)),
\end{array}
$$


where $\psi \in \Psi, r(x, y)$ is defined as in Corollary 14 and $\rho:[0,+\infty) \rightarrow[0,1)$ is a function such that $\rho(t)=0$ if, and only if, $t=0$ and $\lim \sup \rho(t)<1$ for each $s>0$. Then $T$ and $S$ have a unique common fixed point.

$$
t \rightarrow s
$$

Proof. Take $\eta(t, s)=\rho(s) \psi(s)-\psi(t)$ for all $t, s \geq 0$. One can easily show that $\eta$ is a $\Psi$-simulation function. Now the corollary follows from Theorem 3.

Corollary 16. Let $(X, d)$ be a complete metric space and let $T$ and $S$ be two maps on $X$ such that, for every $x, y \in X$,

$$
\begin{aligned}
& \frac{1}{2} \min \{d(x, T x), d(y, S y)\} \leq d(x, y) \quad \text { implies } \\
& \psi((d(T x, S y))+\phi(d(T x, S y)) \leq \psi(r(x, y)),
\end{aligned}
$$

where $\psi \in \Psi, r(x, y)$ is defined as in Corollary 14 and $\phi:[0,+\infty) \rightarrow[0,+\infty)$ is a function such that $\lim \inf \phi(s)>0$ for each $t>0$, and $\phi(t)=0$ if, and only if, $t=0$. Then $T$ and $S$ have a unique common fixed point.

Proof. Take $\eta(t, s)=\psi(s)-\phi(s)-\psi(t)$ for all $t, s \geq 0$. Then similar to the proof of Corollary 13, one can see that $\eta$ is a $\Psi$-simulation function. So, by applying Theorem 3, the proof is completed.

Corollary 17. Let $(X, d)$ be a complete metric space and let $T$ and $S$ be two self-maps on $X$ such that, for every $x, y \in X$,

$$
\begin{gathered}
\frac{1}{2} \min \{d(x, T x), d(y, S y)\} \leq d(x, y) \quad \text { implies } \\
\psi((d(T x, S y)) \leq \phi(\psi(r(x, y)))
\end{gathered}
$$

where $\psi \in \Psi, r(x, y)$ is defined as in Corollary 14 and $\phi:[0,+\infty) \rightarrow[0,+\infty)$ is a function such that, for each $t>0, \phi(t)<t, \lim \sup \phi(s)<t$ and $\phi(t)=0$ if, and only if, $t=0$. Then $T$ and $S$ have a unique common fixed point.

Proof. Define $\eta(t, s)=\phi(\psi(s))-\psi(t)$ for all $t, s \geq 0$. In the proof of Corollary 8 we proved that $\eta$ is a $\Psi$-simulation function. Then we can apply Theorem 3 to complete the proof.

Remark 4. Suppose that $\psi \in \Psi$ and $\phi:[0,+\infty) \rightarrow[0,+\infty)$ is an upper semi-continuous function such that $\phi(t)<t$ for each $t>0$ and $\phi(t)=0$ if, and only if, $t=0$. Then for any sequence $\left\{s_{n}\right\}$ in $(0,+\infty)$ with $\lim _{n \rightarrow \infty} s_{n}=\ell>0$, one can obtain that:

$$
\limsup _{n \rightarrow \infty} \phi\left(\psi\left(s_{n}\right)\right)<\psi(\ell) .
$$

Applying the same argument as in Corollary 17, one can prove the following result.

Corollary 18. Let $(X, d)$ be a complete metric space and let $T$ and $S$ be two self-maps on $X$ such that, for every $x, y \in X$,

$$
\begin{gathered}
\frac{1}{2} \min \{d(x, T x), d(y, S y)\} \leq d(x, y) \quad \text { implies } \\
\psi((d(T x, S y)) \leq \phi(\psi(r(x, y))),
\end{gathered}
$$


where $\psi \in \Psi, r(x, y)$ is defined as in Corollary 14 and $\phi:[0,+\infty) \rightarrow[0,+\infty)$ is an upper semi-continuous function such that $\phi(t)<t$ for each $t>0$, and $\phi(t)=0$ if, and only if, $t=0$. Then $T$ and $S$ have a unique common fixed point.

Definition 4. Let $(X, d)$ be a complete metric space, let $T: X \rightarrow X$ be a mapping and let $\eta \in Z$. Then $T$ is called a generalized $\mathrm{Z}$-contraction with respect to $\eta$ if the following condition is satisfied:

$$
\eta\left(d(T x, T y), r_{T}(x, y)\right) \geq 0,
$$

for all $x, y \in X$, where

$$
r_{T}(x, y)=\max \left\{d(x, y), \frac{[1+d(x, T x)] d(y, T y)}{1+d(x, y)}\right\}
$$

Theorem 7. Every generalized Z-contraction on a complete metric space has a unique fixed point.

Proof. It follows from Corollary 11.

Theorem 8. Let $(X, d)$ be a complete metric space and let $T: X \rightarrow X$ be a continuous map such that, for all $x, y \in X, \frac{1}{2} d(x, T x) \leq d(x, y)$ implies that:

$$
\psi(d(T x, T y)) \leq \psi\left(r_{T}(x, y)\right)-\phi\left(r_{T}(x, y)\right)
$$

where

$$
r_{T}(x, y)=\max \left\{d(x, y), \frac{[1+d(x, T x)] d(y, T y)}{1+d(x, y)}\right\}
$$

and $\psi$ and $\phi$ satisfy:

(i) $\psi:[0,+\infty) \rightarrow[0,+\infty)$ is a continuous non-decreasing function and $\psi(t)=0$ if and only if $t=0$;

(ii) $\phi:[0,+\infty) \rightarrow[0,+\infty)$ is lower semi-continuous with $\phi(t)=0$ if and only if $t=0$.

Then $T$ has a unique fixed point.

Proof. It follows from Corollary 14 when $S=T$.

Theorem 9. Let $(X, d)$ be a complete metric space and $T$ and $S$ be two self-maps on $X$ such that for every $x, y \in X, \frac{1}{2} \min \{d(x, T x), d(y, S y)\} \leq d(x, y)$ implies that

$$
\psi(d(T x, S y)) \leq \psi(r(x, y))-\phi(r(x, y))
$$

where $\psi$ and $\phi$ are given as in Remark 8 and

$$
r(x, y)=\max \left\{d(x, y), \frac{[1+d(x, T x)] d(y, S y)}{1+d(x, y)}\right\} .
$$

Then $T$ and $S$ have a unique common fixed point.

Proof. Since $\phi$ is lower semi-continuous, if $\lim _{n \rightarrow+\infty} S_{n}=\ell>0$, then $\liminf _{n \rightarrow+\infty} \phi\left(S_{n}\right) \geq \phi(\ell)>0$. Therefore, applying Corollary 13, we get the result.

The following example shows that Theorem 3 is a genuine generalization of the Corollary 10.

Example 7. Let $X=\{(0,0,0),(5,5,5),(5,6,5),(5,0,0),(0,0,5)\}$ be endowed with the metric $d$ defined by

$$
d\left(\left(x_{1}, x_{2}, x_{3}\right),\left(y_{1}, y_{2}, y_{3}\right)\right)=\sqrt{\left(x_{1}-y_{1}\right)^{2}+\left(x_{2}-y_{2}\right)^{2}+\left(x_{3}-y_{3}\right)^{2}}
$$


Let $T$ and $S$ be the self-mappings on $X$ defined as follows:

$$
T\left(x_{1}, x_{2}, x_{3}\right)=\left(\min \left\{x_{1}, x_{2}, x_{3}\right\}, 0,0\right),
$$

and

$$
S\left(x_{1}, x_{2}, x_{3}\right)=\left(0,0, \min \left\{x_{1}, x_{2}, x_{3}\right\}\right) .
$$

For any $\eta \in Z_{\Psi}$, the mappings $T$ and $S$ do not satisfy the condition $(46)$ at $x=(5,5,5), y=(5,6,5)$. However, by choosing $\eta(t, s)=\frac{1}{64} s-t$, it is readily verified that $\eta$ is a $\Psi$-simulation function where $\psi$ is the identity function on $[0, \infty)$ and all the hypothesis of Theorem 3 are verified.

To conclude the manuscript, we introduce the following application. Consider the problem

$$
\left\{\begin{array}{l}
f(x(t))=\int_{0}^{t} k(s, x(s)) d s, \quad t \in[0, A] \text { for } A>0, \\
x(0)=0
\end{array}\right.
$$

where $f:[0,+\infty) \rightarrow[0,+\infty)$ and $k:[0, A] \times[0,+\infty) \rightarrow[0,+\infty)$ are continuous mappings. Let $X$ be the family of all continuous functions $x:[0, A] \rightarrow[0,+\infty)$ such that $x(0)=0$ and $x(t) \geq 0$ for all $t>(0, A]$. Define $T, S: X \rightarrow X$ as follows:

$$
T(x)(t)=f(x(t)) \text { and } \quad S(x)(t)=\int_{0}^{t} k(s, x(s)) d s
$$

for al $x \in X$ and all $t \in[0, A]$. Then problem (47) can be seem as the coincidence point problem $T x=S x$ for $x \in X$. Let $d$ be the standard metric on $X$, that is,

$$
d(x, y)=\max (\{|x(t)-y(t)|, t \in[0, A]\}) \quad \text { for all } x, y \in X
$$

Then $(X, d)$ is a complete metric space.

Theorem 10. Consider the problem (47). Assume that $f$ and $k$ are continuous mappings and $f(0)=0$. Assume also that there are $\alpha \in[0,1)$ and $\psi \in \Psi$ such that, for all $x, y \in X$,

$$
\psi\left(\left|f(x(t))-\int_{0}^{t} k(s, x(s)) d s\right|\right) \leq \alpha \psi(m(x, y))
$$

where

$$
m(x, x)=\max _{t \in[0, A]}\left\{\left|f(x(t))-\int_{0}^{t} k(s, x(s)) d s\right|\right\}
$$

and, if $x \neq y$,

$m(x, y)=\max \left\{\max _{t \in[0, A]}|x(t)-y(t)|, \frac{\max _{t \in[0, A]}\{|x(t)-f(x(t))|\} \cdot \max _{t \in[0, A]}\left|y(t)-\int_{0}^{t} k(s, y(s)) d s\right|}{\max _{t \in[0, A]}|x(t)-y(t)|}\right\}$.

Then problem (47) has a unique solution.

Proof. The proof follows from Corollary 1 by taking $\eta(t, s)=\psi(s)-\alpha \psi(t)$ for all $t, s \in[0,+\infty)$.

\section{Conclusions}

The notion of a simulation function is a very interesting tool in fixed point theory that makes it possible to merge and unify several existence fixed point theorems in a single theorem. In this paper, in the setting of a complete metric space, we consider new contractions via the extended simulation 
function by involving two distinct types of rational expressions. It is clear that this approach can be applied to the different abstract structures.

Author Contributions: Investigation, R.A., B.A., E.K. and A.F.R.L.d.H. All authors contributed equally and significantly in writing this article. All authors have read and agreed to the published version of the manuscript Funding: This research received no external funding.

Acknowledgments: The authors thank their universities. The first two authors would like to extend their sincere appreciation to the Deanship of Scientific Research at King Saud University for funding this group No. RG-1437-017. A.F. Roldán López de Hierro is grateful to Project TIN2017-89517-P of Ministerio de Economía, Industria y Competitividad and also to Junta de Andalucía by project FQM-365 of the Andalusian CICYE.

Conflicts of Interest: The authors declare no conflict of interest.

\section{References}

1. Khojasteh, F.; Shukla, S.; Radenović, S. A new approach to the study of fixed point theorems via simulation functions. Filomat 2015, 29, 1189-1194. [CrossRef]

2. Agarwal, R.P.; Karapınar, E. Interpolative Rus-Reich-Ciric Type Contractions Via Simulation Functions, Analele Univ. "Ovidius" Constanta Ser. Mat. 2019, 27, 137-152.

3. Alqahtani, O.; Karapınar, E. A Bilateral Contraction via Simulation Function. Filomat 2019, 33, 4837-4843. doi:10.2298/FIL1915837A. [CrossRef]

4. Aydi, H.; Karapınar, E.; Rakocevic, V. Nonunique Fixed Point Theorems on b-Metric Spaces Via Simulation Functions. Jordan J. Math. Stat. 2019, 12, 265-288.

5. Roldán López de Hierro, A.F.; Karapınar, E.; Roldán López de Hierro, C.; Martínez-Moreno, J. Coincidence point theorems on metric spaces via simulation functions. J. Comput. Appl. Math. 2015, 275, 345-355. [CrossRef]

6. Roldán López de Hierro, A.F.; Shahzad, N. New fixed point theorem under R-contractions. Fixed Point Theory Appl. 2015, Article ID 345. [CrossRef]

7. Roldán López de Hierro, A.F.; Shahzad, N. Fixed point theorems by combining Jleli and Samet's, and Branciari's inequalities. J. Nonlinear Sci. Appl. 2016, 9, 3822-3849.

8. Shahzad, N.; Roldán López de Hierro, A.F.; Khojasteh, F. Some new fixed point theorems under $(\mathcal{A}, S)$-contractivity conditions. RACSAM Rev. R. Acad. A 2017, 111, 307-324.

9. Roldán López de Hierro, A.F.; Shahzad, N. Ample spectrum contractions and related fixed point theorems. Mathematics 2019, 7, 1033. [CrossRef]

10. Alghamdi, M.A.; Gulyaz-Ozyurt, S.; Karapınar, E. A Note on Extended Z-Contraction. Mathematics 2020, 8 , 195. [CrossRef]

11. Roldán López de Hierro, A.F.; Shahzad, N. Common fixed point theorems under $(R, S)$-contractivity conditions. Fixed Point Theory Appl. 2016, Article ID 55, 1-25.

12. Abbas, M.; Lael, F.; Saleem, N. Fuzzy 6 -metric spaces: Fixed point results for $\psi$-contraction correspondences and their application. Axioms 2020, 9, 36. [CrossRef]

13. Rus, I.A. Generalized Contractions and Applications; Cluj University Press: Cluj-Napoca, Romania, 2001.

14. Radenović, S.; Kadelburg, Z.; Jandrlixcx, D.; Jandrlixcx, A. Some results on weak contraction maps. Bull. Iran. Math. Soc. 2012, 38, 625-645.

15. Agarwal, R.P.; Karapınar, E.; O’Regan, D.; Roldán López de Hierro, A.F. Fixed Point Theory in Metric Type Spaces; Springer International Publishing: Cham, Switzerland, 2015.

(C) 2020 by the authors. Licensee MDPI, Basel, Switzerland. This article is an open access article distributed under the terms and conditions of the Creative Commons Attribution (CC BY) license (http://creativecommons.org/licenses/by/4.0/). 